\title{
Arm Relaxation in Deformed H-Polymers in Elongational Flow by SANS
}

\author{
M. Heinrich,* W. Pyckhout-Hintzen, J . Allgaier, and D. Richter \\ Forschungszentrum J ülich, Institut für F estkörperforschung, Postfach 1913, D-52425 J ülich, Germany \\ E. Straube \\ Martin-Luther-Universität HalleWittenberg, Fachbereich Physik, D-06099 Halle, Germany \\ D. J. Read \\ Department of Applied Mathematics, University of Leeds, Leeds, LS2 9J T, U.K.
}

\begin{abstract}
T. C. B. McLeish, D. J . Groves, and R. J . Blackwell
I.R.C. in Polymer Science and Technology, Department of Physics, University of Leeds, Leeds, LS2 9] T, U.K.
\end{abstract}

\author{
A. Wiedenmann \\ Hahn-Meitner Institut, D-14091 Berlin, Germany
}

Received November 5, 2001

\begin{abstract}
We present a small-angl e neutron scattering (SANS) investigation of the relaxation process of branched polymer melts under deformation. The selected model polymer is an $\mathrm{H}$-shaped polyisoprene having deuterium-labeled blocks at the dangling tips of the arms. The melt is step strained in a strain rig especially built for that purpose, where the sample temperature and its deformation conditions are precisely controlled. Uniaxial extensions to values of 2 and 3 are performed at a temperature above the polymer glass transition temperature $T_{g}$. The sample is then all owed to relax during a given time before being quenched to bel ow its $\mathrm{T}_{\mathrm{g}}$ in order to freeze the chains conformation to perform the SANS experiments. Very large anisotropies in $\mathrm{S}(\mathrm{q})$ develop on time scales corresponding to the relaxation of the dangling arms. The experimental structure function is then compared to theoretical ones obtained by the random phase approximation applied to the tube model. While theory describes well the scattering for short times, it seems not to be able to describe the increase of intensity in the direction parallel to the strain at higher times, even when highly nonaffine processes such as arm retraction and branch point withdrawal are taken into account.
\end{abstract}

\section{Introduction}

The past two decades have witnessed a radical change in the way we understand the viscoel astic properties of polymer melts and concentrated solutions. From a standard phenomenological methodology, based on restricted covariant descriptions of the constitutive behavior, a molecular program has now emerged. ${ }^{1}$ Based on the insight that mutually entangled flexible polymer chains move as if they were confined to tubelike constraints al ong their contours, it has immense power since the only parameters required for each chemistry are the tube diameter and monomeric friction constant. The model has successfully accounted for a number of subtle rheological phenomena in linear polymer melts such as the "damping function" in large step shear strains ${ }^{2}$ and the observed $M^{3.4}$ law for the melt viscosity as a function of molecular weight, $M .^{3}$ The additional advantage of a molecular theory for dynamics and rheology is that it can be checked by experiments that probe the molecular conformations directly. In the case of polymer melts the most sensitive technique to the essential conformations of entire chains is small-angle neutron scattering (SANS). ${ }^{4}$ The most direct measurement of the tube diameter, and consequent effect on dynamics, for example, has been made by neutron spin echo (NSE). ${ }^{5}$ The diameter of the tube that results from interpreting the NSE data with the tube model agrees closely with that obtained by intensive numerical simu- lation. ${ }^{6}$ However, the implementation of SANS in the study of highly nonlinear deformations of polymer melts is still in its infancy despite the early progress made by Boué and co-workers in the uniaxial extension of blends of labeled and unlabeled polymers with similar chain lengths. ${ }^{7,8}$ These early experiments aimed to identify, via a change in measured radius of gyration, the prediction due to Doi and Edwards ${ }^{1}$ of a retraction of the chains in the extended tube after a step strain. However, the expected signature of retraction was not observed, and this discrepancy between rheology and scattering remains unexplained. Subsequent experiments (see e.g. refs 9 and 10) examined blends where the labeled species was much shorter than the unlabel ed one, and also networks and gels, all of which gave rise to the anomalous "butterfly" scattering pattern in contours of two-dimensional scattering intensity, where the scattering parallel to the stretch is greatly enhanced down to the smallest wavevectors. A study of partially labeled diblock and triblock copolymers in a high molecular weight unlabeled matrix also gave rise to these patterns. ${ }^{11}$ These patterns appear to be due to elastic inhomogeneities in the entanglement or crosslinked network of the longer chains, ${ }^{12,13}$ which become apparent when the network contains a mobile shorter chain or solvent. In the simplest case of monodisperse linear polymer melts under shear, there is only little published data of single-chain structure factors. ${ }^{7}$ This 
is a pity, because a feature of the molecular approach is that it makes specific and sometimes surprising predictions for the way that unusual rheological response arises via cooperative molecular behavior. This is particularly true of the behavior of long chain branched (LCB) polymer melts. Very recently, a molecular theory for rather generic topologies of LCB polymers was derived from the physics of the tube model. ${ }^{14}$ It explained successfully how the melts can be strongly strain hardening in both planar and uniaxial flows, while retaining strain softening in shear flows. Recently, a polydisperse version of the theory has been used to account quantitatively for a very wide set of nonlinear rheological data on a standard LDPE. ${ }^{15,16}$ To achieve this, it was necessary to invoke a physical consequence of the tube model around the branch points themselves. Termed "branch point withdrawal", it describes the shift of a branch point within a LCB polymer under strain toward the tube containing the strand of greatest tension meeting at the branch point. ${ }^{17}$ In this way, the molecules themselves tend to control the effective friction within their melt environment in a way that turns out to be an essential contribution to their ease of processing. To test the theory, a recent set of experiments on a car efully controlled " $\mathrm{H}$-shaped" architecture polyisoprene melt combined both SANS and nonlinear rheology together with tube theory in an attempt to identify the strong predicted signatures of the effect. ${ }^{18}$ This has required the development of the RPA theory of polymer melts for constraints held out of equilibrium. ${ }^{19}$ The strong anisotropies predicted by the tube and SANS theories combined were actually exceeded by the experimental data!

As an extension to the tube model theory described above, there have been suggested recently several new nonl inear processes for polymer melt rheol ogy. It might be expected that scattering experiments could shed light on these, providing an independent check on the molecular processes which govern melt rheology. The suggested processes include convective constraint release, ${ }^{20-22}$ tube dilation and distortion, ${ }^{23-25}$ nonaffine entanglement deformation from local chain force balance, ${ }^{26}$ and elastic inhomogeneities. ${ }^{12,13}$

In light of this and other scattering studies on deformed melts and solutions ${ }^{12,13}$ that al so show strong heterogeneities under strain, a better controlled series of experiments is vital. In the context of the $\mathrm{H}$-shaped polymer architecture, we need to be able to quench the polymer melt at various stages of its relaxati on to allow a quantitative comparison with the rheological response at each stage. In this paper, we present results of just such an experiment. We compare these results with predictions of the scattering pattern using tube model theory for the various relaxation processes of a stretched melt of $\mathrm{H}$-polymers, coupled with scattering theory based on the random phase approximation. At this stage of modeling we will not attempt to include all of the recently suggested nonlinear effects listed in the previous paragraph, but we will include chain retraction and branch point withdrawal.

The paper is organized as follows. After describing in detail the scattering experiments, we recall the mechanisms of polymer relaxation expected from the tube model. We will describe (mostly in the appendix) the calculation for the scattering from a stretched melt of $\mathrm{H}$-shaped polymers and assess the quality of the prediction in comparison to the experimental results.
A simplification of the scattering function approach valid at early times is presented in anal ogy to a previous treatment of permanent networks. Finally, the tube model theory is critically examined in the light of the experiments. It will be concluded that there remain real problems in the tube model description of the scattering.

\section{Experimental Section}

2.1. Sample Preparation. The $\mathrm{H}$-polymer of 1,4-PI ( $\mathrm{T}_{\mathrm{g}}=$ $-69^{\circ} \mathrm{C}$ ) was prepared in several steps anionically as described in an earlier publication. ${ }^{18}$ The final product is to the largest extent possible of $\mathrm{H}$-architecture whereas lower branching degrees (like stars, for example) cannot be excluded completely. For a similar system, we refer to the specific literature. ${ }^{27}$

The cross-bar (b) is hydrogenous, and the four arms (a) are mainly hydrogenous, but have deuterated dangling tips. Size exclusion chromatography (SEC) and membrane osmometry characterizations gave $M_{n, a}=52500 \mathrm{~g} \mathrm{~mol}^{-1}$ including the deuterated tip of $M_{n, t i p}=10000 \mathrm{~g} \mathrm{~mol}^{-1}$, and $M_{n, b}=111000$ $\mathrm{g} \mathrm{mol}^{-1}$. This leads to a volume fraction of deuterated monomers in the $\mathrm{H}$-molecule $\Phi_{\mathrm{D}}=0.12$.

The sample was mixed to $0.1 \%$ with the antioxidant BHT to avoid chemical degradation or oxidation upon handling. The actual content was determined from UV detection of the antioxidant in SEC batches of varying concentrations of BHT in the running solvent THF. Stripes of samples of length 45 $\mathrm{mm}$, width $10 \mathrm{~mm}$, and thickness $1 \mathrm{~mm}$ were obtained from vacuum molding at a temperature of $55^{\circ} \mathrm{C}$ for approximately $5 \mathrm{~h}$. Upper and lower parts were reinforced to enhance the grip on the clamps of the strain rig.

2.2. Rheological Characterization. As a check of the nonlinear dynamical behavior of the $\mathrm{H}$-polymer melt and of the accuracy of the molecular theory at high strains, the polymer was measured in a series of transient shear flows at varying rates as well as in linear oscillating shear. All experiments were performed on a Rheometrics RDAll rheometer, using parallel plates for the oscillatory measurements and a cone and plate for the transient flow.

Linear rheological measurements were made at a range of temperatures from -30 to $90^{\circ} \mathrm{C}$. The obtained moduli $\mathrm{G}^{\prime}(\omega)$ and $\mathrm{G}^{\prime \prime}(\omega)$ were shifted to a reference temperature of $\mathrm{T}_{0}=25$ ${ }^{\circ} \mathrm{C}$, using the WLF time-temperature superposition principle. The corresponding coefficients were $C_{1}=4.86$ and $C_{2}=138.1$. The results are shown in Figure $1 \mathrm{~A}$ al ong with a theoretical fit from a tube model calculation. ${ }^{18}$ This permits a robust assignment of the sections of arms that have orientationally relaxed at the annealing stages in the neutron scattering experiment and shown in Figure 2.

The transient measurements in nonlinear shear flow were made at $90^{\circ} \mathrm{C}$, and the results are shown in Figure 1B. Again, the results are compared with a tube model calculation, which provides a consistency check for the tube model parameters (including the degree of branch point withdrawal) as discussed in ref 18. The reason for this nonlinear rheological experiment was to provide an independent measure to the SANS of the response of the $\mathrm{H}$-melt to strains that would stretch the crossbar sections of the molecules (albeit temporarily). The shear stress overshoots could be accounted for by mild branch point withdrawal.

2.3. Strain Rig. A strain rig with appropriate temperature as well as strain rate control was built at FZJ ülich (see Figure 3). It consists of a mechanical testing unit and a temperature control unit. The lower cylinder is static whereas the upper piston may be driven up by a step motor with a maximum load of $300 \mathrm{~N}$ and speeds between $10^{-4}$ and $75 \mathrm{~mm} \mathrm{~s}^{-1}$ to give a strain rate range between $4 \times 10^{-7}$ and $4 \mathrm{~s}^{-1}$. The constant strain rate condition is fulfilled by exponentially increasing the crosshead speed with time as $v(t)=\dot{\epsilon} \mathrm{L}_{0} \exp (\dot{\epsilon} \mathrm{t})$ corresponding to an increase in the sample length as $L(t)=L_{0} \exp (\epsilon t)$. A smooth profile is achieved by subdividing into 3000 discrete intervals to realize a nearly continuous deformation.

The temperature of the sample can be varied between -140 and $+200{ }^{\circ} \mathrm{C}$ with an accuracy of $\pm 0.1^{\circ} \mathrm{C}$ and is monitored 

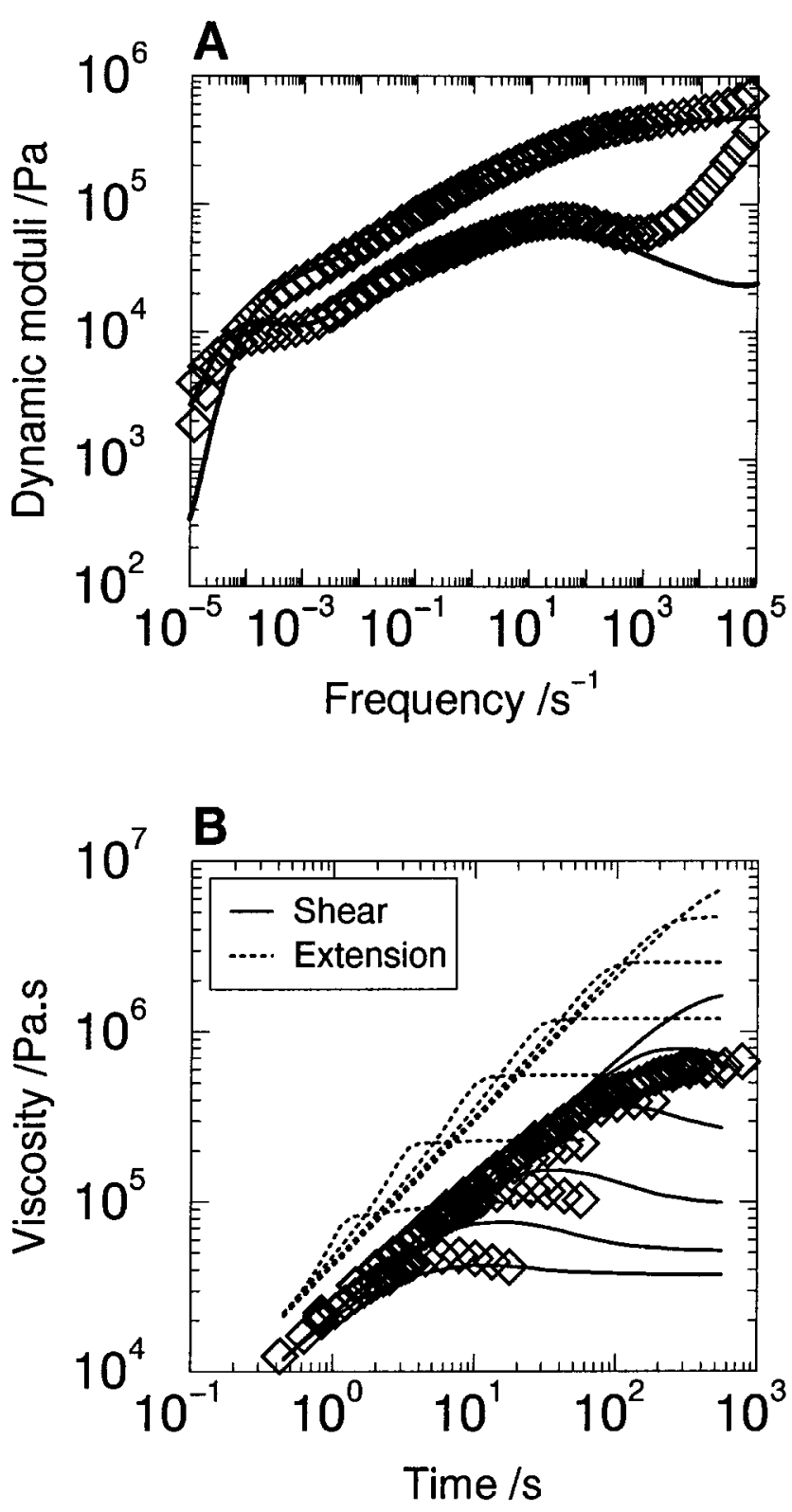

Figure 1. (A) Experimental dynamic moduli $\mathrm{G}^{\prime}(\omega)$ and $\mathrm{G}^{\prime \prime}$ $(\omega)$ (diamonds) in oscillatory shear at $25^{\circ} \mathrm{C}$ compared with a theoretical fit from a tube model calculation (lines). (B) Transient measurements in nonlinear shear flow (diamonds) made at $90^{\circ} \mathrm{C}$ compared with a tube model calculation (lines). Rates are $0.003,0.01,0.03,0.1,0.3$, and $1 \mathrm{~s}^{-1}$ (from top to bottom), and a tube model calculation for uniaxial extension is also shown.

during the experiment. The cooling and heating medium is liquid $\mathrm{N}_{2}$, whereas for temperatures higher than the ambient temperature also gaseous $\mathrm{N}_{2}$ can be employed. This enables quench experiments as well as crystallization studies. Pollution or penetration of silicone oil in the sample which cannot be excluded in the setup of ref 28 is avoided here as a main advantage.

The thermobox consists of three parts:

In the lower part of the thermobox, a "heating spiral" (maximum power $600 \mathrm{~W}$ ) heats a brass oven to the desired temperature. By means of evaporated carrier gas $\mathrm{N}_{2}$, this heat is conducted onto the sample through four diffusers. The consumption of $\mathrm{N}_{2}$ is of the order of $5 \mathrm{~L} / \mathrm{h}$ and precisely controlled using an adapted He tap, so that the desired temperature $T_{0}$ is easily reached and maintained.

The middl e section contains the sample and mainly consists of a neutron transparent quartz cell with double walls. Inside

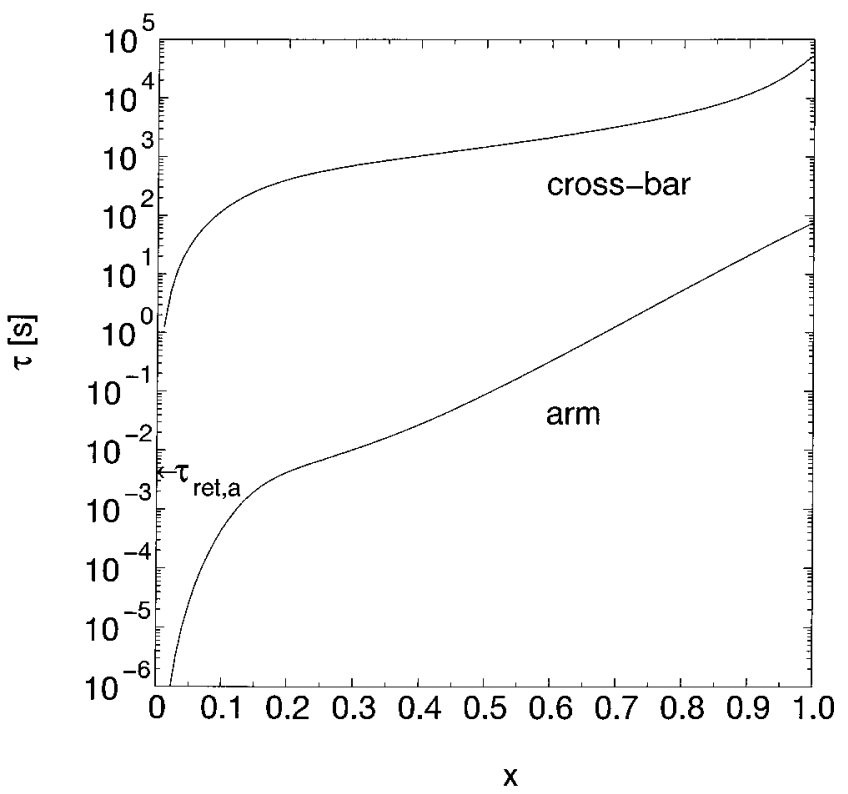

Figure 2. Theoretical annealing time $\tau$ as a function of $x$, the abscissa al ong the chain ( $x=0$ at the branch point) for the arm and the cross-bar obtained from linear measurements in oscillatory shear (see Figure 1).

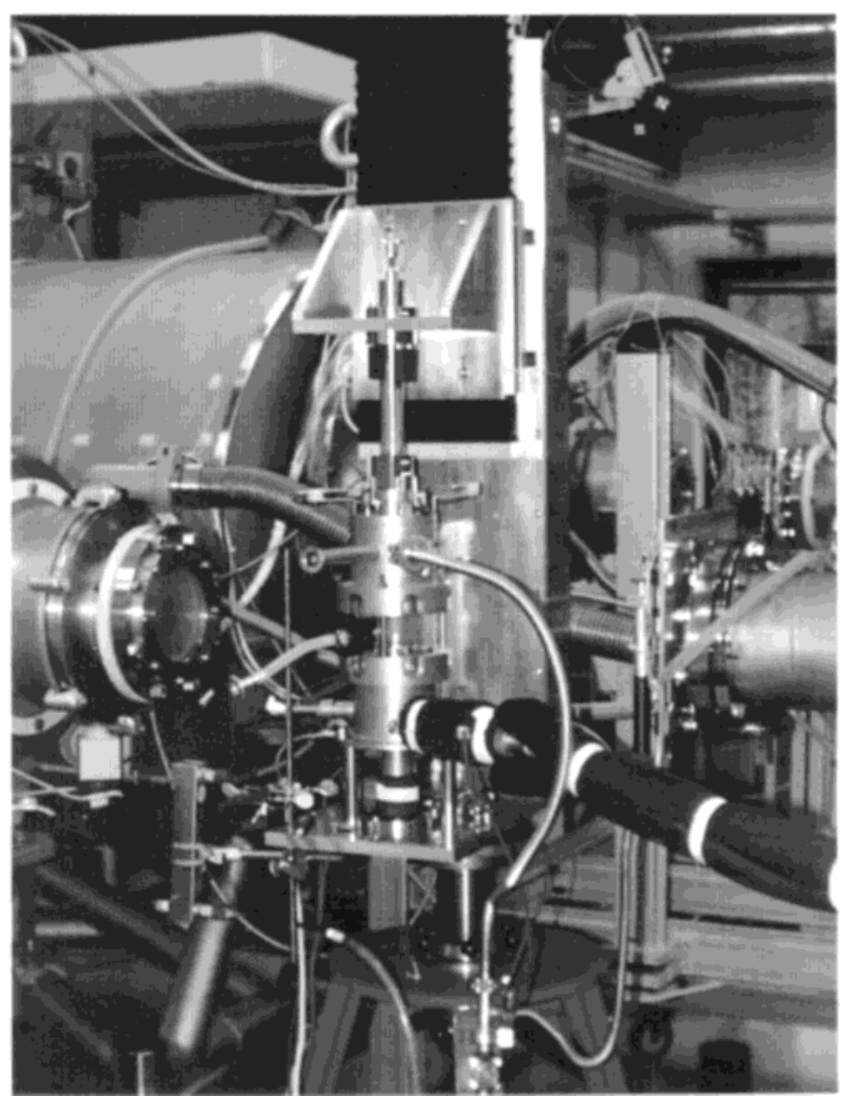

Figure 3. Strain rig with temperature and strain rate control in the neutron beam at KWS1.

the cell walls, a vacuum of about $10^{-6}$ mbar is kept to ensure a good isolation and to minimize water condensation which would hinder the neutron beam passing through and scatter parasitically and avoids uncontrolled temperature losses as well. By an inlet at mid-height of this cell a high flux of liquid $\mathrm{N}_{2}$ can be directed straight on the deformed sample when quenching is needed. With this cell, the straining can be done in situ in the neutron beam for low- $T_{g}$ materials. 
In the upper part, four thermocouples ( $\mathrm{Fe}-\mathrm{Cu}$ ) measure the temperature in the immediate vicinity of the sample. It was assured that the temperature of the gas is the same as that of the sample. This temperature is fed back to the spiral heater. The heating rate of about $5{ }^{\circ} \mathrm{C} / \mathrm{min}$ avoids overshoots which affect chain relaxation drastically. The gaseous $\mathrm{N}_{2}$ leaves the thermobox through an outlet in its upper part.

The lower and the upper parts of the thermobox are evacuated during the experiment for isolation purposes and homogeneous temperature distribution in the thermobox.

For temperature stability and friction reasons, the gap between shaft and thermobox is about $0.2 \mathrm{~mm}$.

2.4. SANS Experiments. SANS experiments were performed at the $\mathrm{V} 4$ instrument at the $\mathrm{HMI}$, Berlin, with neutron wavelength $\lambda_{N}=8 \AA$ and $\Delta \lambda_{N} / \lambda_{N}=11 \%$ and at $\mathrm{KWS1}$ in $\mathrm{FZ}$ J ülich at $\lambda_{N}=7 \AA$ with a relative dispersion $\Delta \lambda_{N} / \lambda_{N}=20 \%$.

Two different sample-detector distances ( 2 and $7 \mathrm{~m}$ at V4 and 2 and $8 \mathrm{~m}$ at KWS1) were necessary to cover the scattering vector range $7 \times 10^{-3}<\mathrm{q}\left(\AA^{-1}\right)<10^{-1}$, the scattering vector being defined as $q=4 \pi \sin (\theta / 2) / \lambda_{\mathrm{N}}$. Two-dimensional scattering data were corrected pixel wise in the standard way for sensitivity, empty cell scattering, dark current, and absolutely calibrated by means of water or lupolene calibration standard. The quality of the overlap can be seen in the principal axis data, which were obtained from a procedure, thereby eliminating systematic errors upon regrouping angularly. The procedure was described earlier. ${ }^{29}$

Based on the linear viscoelasticity and assuming simple thermorheological behavior for uniaxial deformation to focus on the time scale of relaxation of the faster arm processes, the sample was uniaxially stretched at $-30{ }^{\circ} \mathrm{C}$ in $5 \mathrm{~s}$ from its initial length $L_{0}=35 \mathrm{~mm}$ to a length $\mathrm{L}=70 \mathrm{~mm}$ (corresponding to a strain $\lambda=2$ ) or in $8 \mathrm{~s}$ from its initial length $\mathrm{L}_{0}=35$ $\mathrm{mm}$ to a length $\mathrm{L}=105 \mathrm{~mm}$ (corresponding to a strain $\lambda=3$ ), with a constant strain rate $\dot{\epsilon}=1.386 \times 10^{-1} \mathrm{~s}^{-1}$ and then quenched to $-85^{\circ} \mathrm{C}$ within about $3-5 \mathrm{~s}$. Quenching below the sample glass transition temperature is needed before each SANS experiment in order to freeze in the chain conformation and thus avoid the chain relaxation during the scattering measurement (typically about $7 \mathrm{~h}$ ). The stretching itself was performed at $\mathrm{T}_{\mathrm{g}}+40^{\circ} \mathrm{C}$, where the deformation is as cl ose as possible to instantaneous with respect to the molecular relaxation processes of the polymer chains.

The SANS experiments were performed at $-85 \pm 1{ }^{\circ} \mathrm{C}$. Relaxation processes could be restarted by raising the temperature of the system again over $\mathrm{T}_{\mathrm{g}}$ for controlled periods.

In this way, it was possible to study the structure of the melt after different annealing times. The sketch of the experimental procedure is given in Figure 4.

The time necessary for the sample to reach its final relaxation temperature (e.g., $-30^{\circ} \mathrm{C}$ in this example) starting from $-85^{\circ} \mathrm{C}$ was taken into account in the determination of the effective time of relaxation. On the other hand, the quenching time (less than $5 \mathrm{~s}$ ) can be safely neglected compared to the total relaxation time.

As a consequence, the sample relaxes at several temperatures during one annealing time. For consistency, the times measured at these different temperatures were converted to $25^{\circ} \mathrm{C}$ using the WLF time-temperature shift factors. ${ }^{30} \mathrm{~A}$ given time $t_{T}$ measured at temperature $T$ is then converted to a time $t_{T_{0}}$ at a temperature $T_{0}$ as $t_{T_{0}}=t_{T} / a_{T \rightarrow T_{0}}$. These shift factors were determined independently from rheology by $\log \mathrm{a}_{\mathrm{T}_{\rightarrow} \mathrm{T}_{0}}=$ $-\mathrm{C}_{1}\left(\mathrm{~T}-\mathrm{T}_{0}\right) /\left(\mathrm{C}_{2}+\mathrm{T}-\mathrm{T}_{0}\right)$.

The effective time of relaxation $\tau$ is obtained by summing the time due to the sample heating ramp to the time where the sample is effectively at $-30^{\circ} \mathrm{C}$. Let $\tau_{1}$ be the time the sample needs to reach $-30{ }^{\circ} \mathrm{C}$ and $\mathrm{T}(\mathrm{t})$ its temperature as a function of time during the heating ramp and $\tau_{2}$ the time at

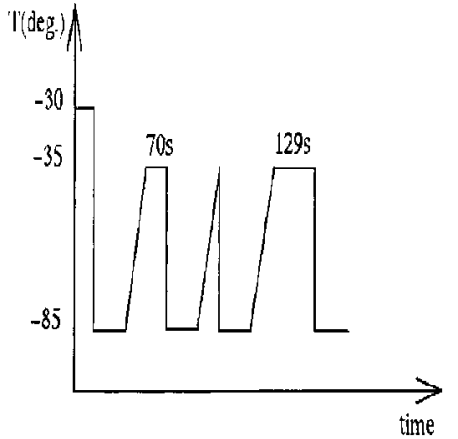

a

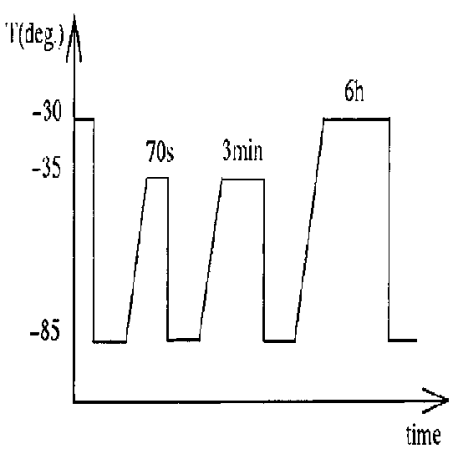

$\mathrm{b}$

Figure 4. Temperature history of the sample, after strains at $-30{ }^{\circ} \mathrm{C}$ to (a) $\lambda=2$ and (b) $\lambda=3$. In each case, SANS experiments are performed at $-85^{\circ} \mathrm{C}$, after annealing times equivalent to: (a) " 0 ", $2.3 \times 10^{-2}, 3.0 \times 10^{-2}$, and $6.6 \times 10^{-2}$ s at $25^{\circ} \mathrm{C}$; (b) " 0 ", $2.3 \times 10^{-2}, 8.7 \times 10^{-2}$, and $13 \mathrm{~s}$ at $25^{\circ} \mathrm{C}$.

which the sample is effectively at $-30^{\circ} \mathrm{C}$, then the total time of relaxation $\tau$ at $25^{\circ} \mathrm{C}$ is

$$
\tau=\int_{0}^{\tau_{1}} \frac{\mathrm{dt}}{\mathrm{a}_{\mathrm{T}(\mathrm{t}) \rightarrow 25}}+\frac{\tau_{2}}{\mathrm{a}_{-30 \rightarrow 25}}
$$

For all the SANS experiments performed, the effective times of relaxation shifted to $25^{\circ} \mathrm{C}$ are summarized in the legend of Figure 4. The total accuracy of times, estimated from error propagation taking into account $\Delta \mathrm{C}_{1}$ and $\Delta \mathrm{C}_{2}$, the uncertainty of the sample temperature and the uncertainty of the temperature ramp, is about $50 \%$ and sufficient for comparison of annealing times during the SANS experiments and the time constants from linear rheology.

Exact homogeneity in the sample deformation was observed for test specimen (PS melt and PI network), which is an indirect but important and necessary proof of the temperature profile of the sample.

\section{Rheological Relaxation Processes}

Rheological measurements, together with theoretical modeling using the tube model, have al ready identified a set of relaxation processes for $\mathrm{H}$-shaped polymers, and given the time scales for each stage of the relaxation. ${ }^{18}$ This knowledge is necessary to estimate the nonlinear response in this strain experiment. We will summarize the basic times and provide the expected amount of relaxing segments as a function of the annealing time in this transient experiment. The times given will be based on the characteristic data for the $\mathrm{H}$-polymer in this investigation and obtained from a fitting to the dynamic moduli.

The linear response is mainly described by three processes: the first two belong to the arm relaxation domain; a rapid equilibration of tip ends by a Rouse- 
like longitudinal relaxation, followed by an activated process of contour length fluctuations. For the $\mathrm{H}$-arm in this study the time scale of relaxation in linear (small amplitude) rheology extends from $10^{-5}$ to about $100 \mathrm{~s}$ at $300 \mathrm{~K}$. This is well inside the experimental ly accessed time regime in the nonlinear strain experiment. The third and slowest process is given by the contour length fluctuation and reptation of the cross-bar chain in an effective tube defined only by other cross-bars. The relaxed arms provide the effective friction centers at the ends of the quasi-linear cross-bar. The relaxation time spectrum of the cross-bar is situated between 10 and 5 $\times 10^{5} \mathrm{~s}$ and is dominated by reptation at the longest time scale. The cross-bar relaxation is well separated from the arm relaxation in time (see also Figure 2).

In the loss modulus curve (Figure $1 \mathrm{~A}$ ) the arm relaxation corresponds to the broad "shoulder" toward the high-frequency end of the spectrum, while the crossbar relaxation (mainly via reptation) corresponds to the low-frequency peak. The theoretical fit uses the calculation described in ref 18 , and the calculated relaxation spectrum agrees well with the measured modulus when arm polydispersity is taken into account (solid curves). The sole four parameters for a consistent description are the Rouse time for one entanglement length $\tau_{\mathrm{e}} \approx$ $10^{-5} \mathrm{~s}$ at $300 \mathrm{~K}$, the plateau modulus $\mathrm{G}_{0}=0.48 \mathrm{MPa}$, the number of entanglement spacings al ong the arm and cross-bar given as $\mathrm{M}_{\mathrm{a}} / \mathrm{M}_{\mathrm{e}}=12$ and $\mathrm{M}_{\mathrm{b}} / \mathrm{M}_{\mathrm{e}}=26$, respectively, and their corresponding relative volume fractions. $M_{e}$ is the entanglement molecular weight corresponding to the mass of a chain that traverses one tube diameter.

As part of the theoretical description of the linear rheol ogy, a process known as tube dilation is considered. During the relaxation, the number of active entanglements to the topology is reduced, and the relaxed arms act as an effective solvent for the remaining entangled polymers. (A relaxed arm no longer provides topol ogical constraints.) This process of "tube dilation" through constraint release occurs concurrently with the arm relaxation and affects the tube diameter, which scales as

$$
d_{0}=\frac{d_{00}}{\left(1-x\left(\frac{4 M_{a}}{M_{\text {tot }}}\right)\right)^{\alpha / 2}}
$$

Here, $x$ is the fraction of relaxed segments in the arms, $M_{a}$ and $M_{\text {tot }}$ are the molecular weight of an arm and the total molecular weight of the molecule, and $d_{00}$ is the undiluted value for the end-to-end distance of entanglements. For the dilution exponent $\alpha$ the scaling result $\alpha=4 / 3$ is used as in ref 18 , but our results are not strongly affected by other choices in the vicinity of $\alpha \approx 1$.

In a nonlinear stretch experiment, however, the number of relaxation modes is increased. This is due to the fact that, after a nonlinear strain, the length of the tube is increased, and the chain finds relaxation pathways towards its equilibrium length within the tube. One possible pathway is via curvilinear motion of the chain along the tube contour. The chains within the tubes are initially stretched beyond their equilibrium tube length, and there is an entropic gain in retracting within the tube to the equilibrium tube length. For the $\mathrm{H}$-polymer arms, such a retraction along the tube is unimpeded, since it requires only the pulling of a chain free end into the tube. It is al so rel atively fast; the time scale for arm retraction is just the Rouse time for curvilinear motion of the arms al ong the tube contour, subject to one end being tethered. The estimated time is $\tau_{\text {ret, } a}=4 \tau_{\mathrm{R}} \approx 0.004 \mathrm{~s}$ and indicated in Figure 2 .

For the $\mathrm{H}$-polymer cross-bar, however, a rapid retraction along the tube contour is impeded by the branch points and involves pulling the arms into the cross-bar tube. For this there is an entropy penalty. The competition between both entropy gain and entropy penalty was resolved by a "force balance" argument. ${ }^{31}$ The chain tension in the tube for the cross-bar must be greater than the sum of the (equilibrium) chain tensions in the two arms for retraction to occur significantly. This requires that the cross-bar must be stretched to twice its equilibrium tube contour length, requiring a uniaxial stretch of the melt of order $\lambda=4$. Nevertheless, it is possible to speculate that some cross-bar retraction occurs earlier than this threshold, due to local displacement of the branch point within its cage of entanglements. Also, some chains will achieve a tension twice that of equilibrium before $\lambda=4$, due to preferential orientation in the direction of principal strain. Theoretical models of polymer rheology which assume such a partial branch point withdrawal have indeed produced an improved description of branched polymer rheology. ${ }^{16}$ This branch point withdrawal is also conjectured to be partially responsible for observed SANS anisotropy. ${ }^{18}$

The purpose of the nonlinear shear experiment was to provide some check on the degree of this motion of the branch point when the cross-bar suffers higher stretch than the arms. The motion of the branch point under strain is governed within the theoretical model by the parameter $v^{*}$ (for details of the calculation, see ref 16). When modeling nonlinear rheology, there is sometimes a discrepancy between parameters used to fit transient shear and extensional data. However, in the tube model theory we have employed (see ref 18 for details), after fitting the dynamic moduli only one additional parameter is required: $v^{*}$. This parameter controls the maximum in the transient stress growth in both shear and extension, through the degree of branch point withdrawal. While the effect of $v^{*}$ is more pronounced in extension than shear, the limited amount of $\mathrm{H}$-polymer available means that we could only perform nonlinear shear experiments. In transient shear experiments, the parameter $v^{*}$ controls the shape and extent of the shear stress maximum observed at high shear rates and early times. In the curves shown in Figure $1 \mathrm{~B}$, the value of $v^{*}$ is consistent with other polymers ${ }^{18}$ and with the shifts assumed in analyzing the SANS data. The rheology is consistent with the assumption of sufficient tube-diameter displacement of the branch point in the extensional strains imposed during the SANS experiments, to strongly modify the stretch relaxation rate.

The transient shear response shown in Figure 1B is broadly captured at most shear rates, as it was for a smaller polymer reported in ref 18 . The location and the shape of the transient peaks are captured in the theory. However, the precise onset of shear thinning does show some discrepancy between theory and experiment. Departures of this kind are unsurprising since the relaxation times in the theory were chosen to fit the linear viscoel astic measurements in Figure 1A. Nevertheless, it is clear that the tube model can capture the linear viscoelastic behavior and that the model is 
consistent with the transient shear experiments in Figure 1B. Theoretical predictions for nonlinear extension at $90{ }^{\circ} \mathrm{C}$ are also shown in Figure 1B; these are similar to experimental results on a smaller $\mathrm{H}$-polymer. ${ }^{18}$ This demonstrates the extent of the extension hardening that is consistent with the tube model and the other rheol ogical data.

To the extent that it can happen, cross-bar retraction with branch point withdrawal is a fast process, requiring only curvilinear motion of the chain along the tube contour. It occurs on a time scale of order of the Rouse time for the whole $\mathrm{H}$-molecule, which we estimate to be $0.04 \mathrm{~s}$.

The force balance argument of McL eish indicates that cross-bar retraction via branch point withdrawal al one can never return the cross-bar to its equilibrium length. The terminal relaxation of the cross-bar stretch occurs on time scales larger than the longest activated contour length fluctuation of the arms; at such time scales, the arms act as concentrated centers of friction. The terminal stretch time of the cross-bar is given by a balance between the entropic restoring force in the cross-bar and the friction due to the arms, as detailed in ref 18 . In the present case, we estimate this to be about $2240 \mathrm{~s}$.

The following ideal scenario is under investigation: before stretching, the polymers are, on average, distributed isotropically. If the melt is now strained sufficiently rapidly as to avoid any of the above relaxation processes, each monomer in the melt is convected affinely with the strain, and the polymer chains as a whole become oriented at all length scales. Because the chains were convected affinely with the strain, the melt topology, and hence the tube contour, will also be deformed affinely with the strain. The "tubes" felt by the polymers at this time will hence be the same tubes that were present in the isotropic melt just before the stretch, but affinely deformed. An assumption of the tube model is that these tubes continue to be fixed in space-in their affinely deformed positions-for the duration of the polymer relaxation (i.e., until a given tube segment is "relaxed" by a chain end passing through it). This leads to the tube dilution picture given above. For times later than the Rouse time of an entanglement segment, the chains relax subject to the constraints imposed by the tubes: relaxation becomes a problem of "escape from the oriented tube segments" as in linear rheology but now with the additional relaxation processes of chain withdrawal or retraction. We suppose that the "linear" and "nonlinear" relaxation processes occur simultaneously.

In the experiment, however, the time of stretching was $5 \mathrm{~s}$ (or $8 \mathrm{~s}$ ) at $-30^{\circ} \mathrm{C}$, which roughly corresponds to $3 \times 10^{-3} \mathrm{~s}$ (or $4.8 \times 10^{-3} \mathrm{~s}$ ) at $25^{\circ} \mathrm{C}$ using the shift factors of linear rheology. This is not "instantaneous" when compared to some of the relaxation processes above but is of the same order as the Rouse stretch relaxation time of the arm and of the orientational relaxation time for the arm tips. Therefore, we would expect only a partial stretching of arms and no orientation of the labeled tips. From Figure 2 it can be seen that, in order to observe considerable cross-bar relaxation processes, relaxation times of the order of $100 \mathrm{~s}$ at $25^{\circ} \mathrm{C}$, i.e., $10^{5} \mathrm{~s}$ at $-30^{\circ} \mathrm{C}$, would have been required. In this experiment, therefore, the cross-bar is well fixed in time. We access the earlier relaxation processes but not the later ones.
Table 1. Diluted Tube Diameter do, Fraction of Relaxed Arm x Obtained from SANS for Both Theoretical Approaches ( $q$ = "Quenched-RPA", $f=$ "F actorized-RPA") and $x$ (th.rheo), Fraction of Relaxed Arm Obtained from Theory Applied to Rheology ${ }^{16}$ for the Strains $\lambda=2$ and 3 after Annealing Times $\tau$ at $25^{\circ} \mathrm{C}$

\begin{tabular}{llllllc}
\hline$\lambda$ & \multicolumn{1}{c}{$\tau$ at $25^{\circ} \mathrm{C}(\mathrm{s})$} & $\mathrm{x}(\mathrm{q})^{\mathrm{a}}$ & $\begin{array}{c}\mathrm{d}_{0}(\AA) \\
(\mathrm{q})\end{array}$ & $\mathrm{x}(\mathrm{f})^{\mathrm{a}}$ & $\begin{array}{c}\mathrm{d}_{0}(\AA) \\
(\mathrm{f})\end{array}$ & $\begin{array}{c}\mathrm{x} \\
(\text { th.rheo })\end{array}$ \\
\hline 2 & ${ }^{\prime \prime} \mathrm{O}^{\prime \prime b}$ & 0.025 & 31 & 0 & 32 & 0.12 \\
2 & $(2.3 \pm 1.1) \times 10^{-2}$ & 0.086 & 32 & 0.078 & 33 & 0.38 \\
2 & $(3.0 \pm 1.5) \times 10^{-2}$ & 0.092 & 32 & 0.091 & 33 & 0.41 \\
2 & $(6.6 \pm 3.3) \times 10^{-2}$ & 0.116 & 33 & 0.118 & 34 & 0.48 \\
3 & ${ }^{\prime \prime} 0^{\prime \prime b}$ & 0.025 & 31 & 0.038 & 32 & 0.12 \\
$3^{c}$ & $(2.3 \pm 1.1) \times 10^{-2}$ & 0.147 & 36 & 0.14 & 34 & 0.38 \\
$3^{\mathrm{d}}$ & $(8.7 \pm 4.3) \times 10^{-2}$ & 0.31 & 44 & n.f.e & n.f. & 0.50 \\
$3^{\mathrm{d}}$ & $13 \pm 6$ & 0.40 & 46 & n.f. & n.f. & 0.86
\end{tabular}

a The uncertainties in $x$ are estimated to be 0.02 absolute; the ones in $\mathrm{d}_{0}$ are estimated to be $1 \AA$. ' about $10^{-3} \mathrm{~S} .{ }^{c}$ Additional full arm retraction is taken into account to describe these data for the "quenched-RPA" approach. ${ }^{d}$ Additional full arm retraction and some cross-bar retraction are taken into account to describe these data, but we do not consider that the corresponding parameters $x(q)$ and $d_{0}(q)$ are the true parameters as the fits are poor. ${ }^{e}$ n.f. $=$ nonfittable.

In light of these predictions, the scattering experiment should be ideally suited for an independent detection of the relaxation processes. It will be a sensitive test of the validity of transferring linear information into highly nonlinear conditions, a purpose which is of particular interest to those needing to predict the properties of industrial polymers from their linear behavior.

\section{Initial Consideration of the Scattering Data}

4.1. $\lambda=2$ and Relaxation. The temperature history of the nonlinear step-strain experiment is summarized in Figure 4 and discussed in more detail in the Experimental Section together with Table 1. From the linear rheology, the annealing times chosen should cover a large part of the arm relaxation spectrum (up to about halfly relaxed the arms).

The corresponding two-dimensional data at $\lambda=2$ are presented for both detector distances in Figure 5, where the vertical axis is the strain direction. It shows clearly how anisotropy evolves with time. The length scale of anisotropy depends on the annealing time. In particular, we notice that the scattering peak is highest in parallel direction and growing in time (note that the $\lambda=2$ data do not cover the later times in the $\lambda=3$ data presented below). As time increases, the scattering at large wavevectors becomes more isotropic, indicating that at small length scales the polymer is effectively relaxed (by relaxation within the tube and through the arm breathing modes). Note that the expected position of the peak perpendicular to the stretch (from a simple affine deformation of the isotropic scattering peak of the unstretched sample) lies within this "large wavevector" region. The perpendicular peak is effectively "washed out" by the relaxation of the polymer at small length scales.

4.2. $\lambda=3$ and Relaxation. To investigate the relaxation dependence on strain, the stretching time was adapted to keep the strain rate constant and not to introduce a new variable. Figure 6 shows a collection of two-dimensional data at the annealing times " 0 ", 2.3 $\times 10^{-2}, 8.7 \times 10^{-2}$, and $13 \mathrm{~s}$ at $25^{\circ} \mathrm{C}$ and $\lambda=3$. Note that the latter two annealing times were both larger than those probed in the $\lambda=2$ data. The anisotropy is 


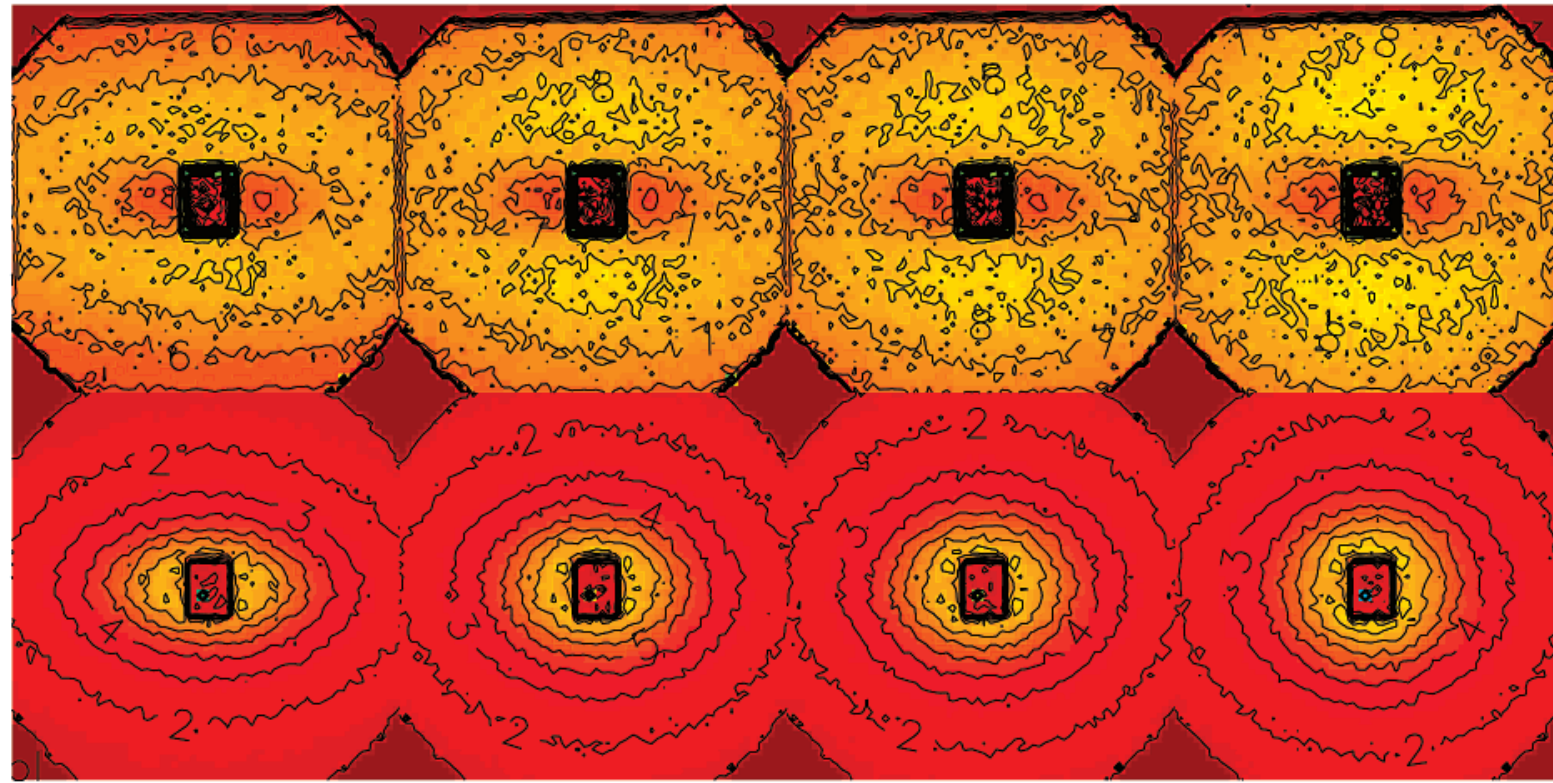
"O" s
$2.310^{-2} \mathrm{~s}$
$310^{-2} \mathrm{~s}$
$6.610^{-2} \mathrm{~s}$

Figure 5. Two-dimensional SANS data for the sample strained to $\lambda=2$, for the low-q limit (top) and high-q limit (bottom), the vertical axis being the strain direction. From the left to the right: "0", $2.3 \times 10^{-2}, 3.0 \times 10^{-2}$, and $6.6 \times 10^{-2} \mathrm{~s}$ relaxation at 25 ${ }^{\circ} \mathrm{C}$.

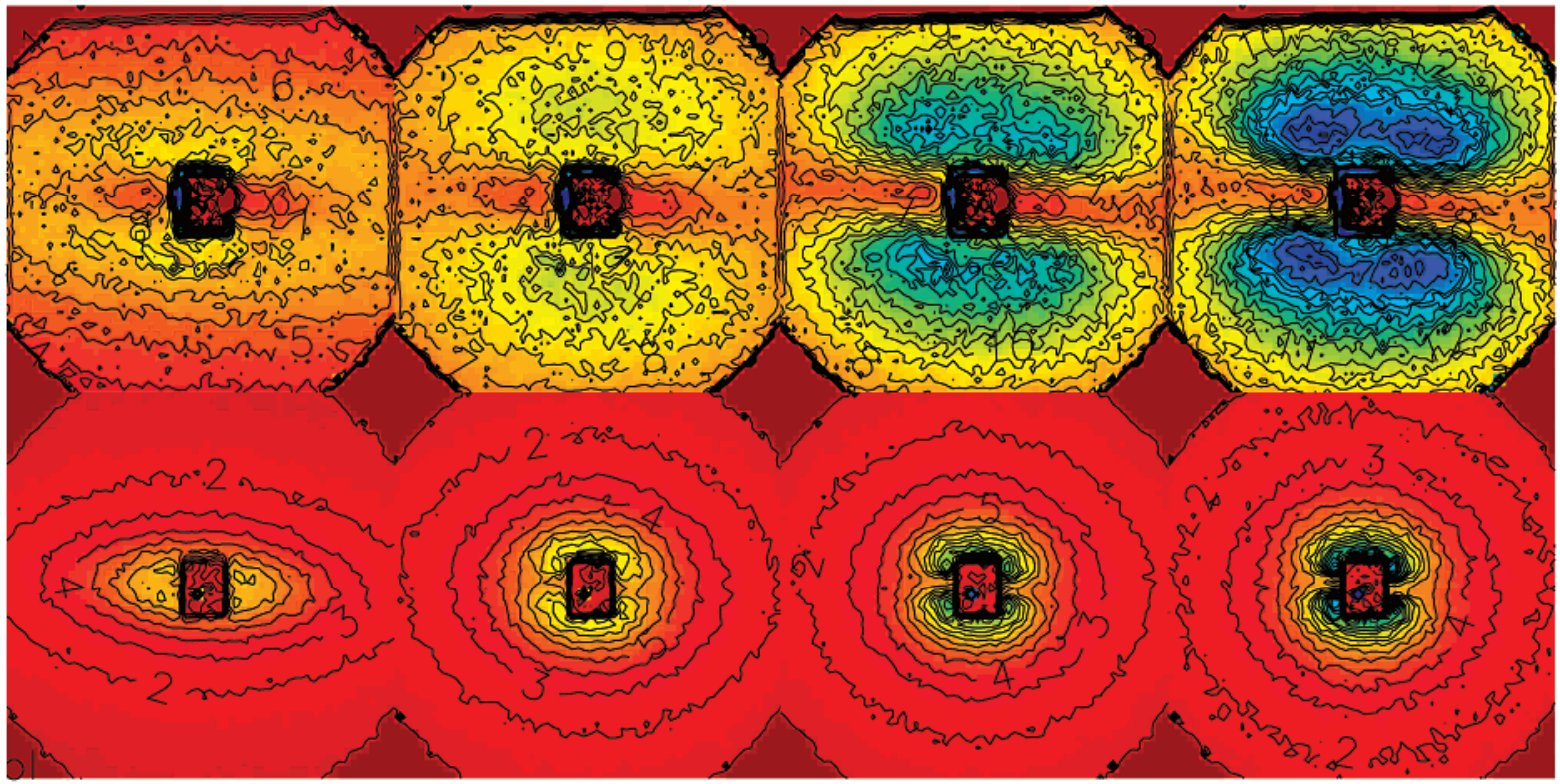
"O" s
$2.310^{-2} \mathrm{~s}$
$8.710^{-2} \mathrm{~s}$
$13 \mathrm{~s}$

Figure 6. Two-dimensional SANS data for the sample strained to $\lambda=3$, for the low-q limit (top) and high-q limit (bottom), the vertical axis being the strain direction. From the left to the right: " 0 ", $2.3 \times 10^{-2}, 8.7 \times 10^{-2}$, and 13 s relaxation at $25^{\circ} \mathrm{C}$. The occurrence of extra four off-axis peaks is seen in the low-q limit for the two highest relaxation times.

more pronounced than in the $\lambda=2$ data. With evolving time, considerable isotropy is regained in the high-q limit (lower data of Figure 6). For lower scattering vectors, the peak parallel to the stretch is much larger than that perpendicular to the stretch, and it is quite clear that the parallel peak is growing as the relaxation time increases. In other words, the scattering pattern in the low-q region gets more anisotropic as the stress relaxes! This is highly counterintuitive. This increasing low-q anisotropy occurs well after the full relaxation of the initial anisotropy at high-q. Although these observa- tions are reminiscent of observations of "butterfly" or "8-shaped" patterns in scattering from asymmetric blends and gels (see e.g. refs 9 and 10), they are distinctly different. In the present case, the scattering retains the "correlation hole" structure of a block copolymer melt; it is still peaked at finite wavevector and tends toward zero scattering at the origin. This is not a feature of the usual butterfly patterns, where the anisotropy persists to the "peak" at zero wavevector. Nevertheless, we cannot rule out the possibility that at least some of the anisotropy seen here has the same 

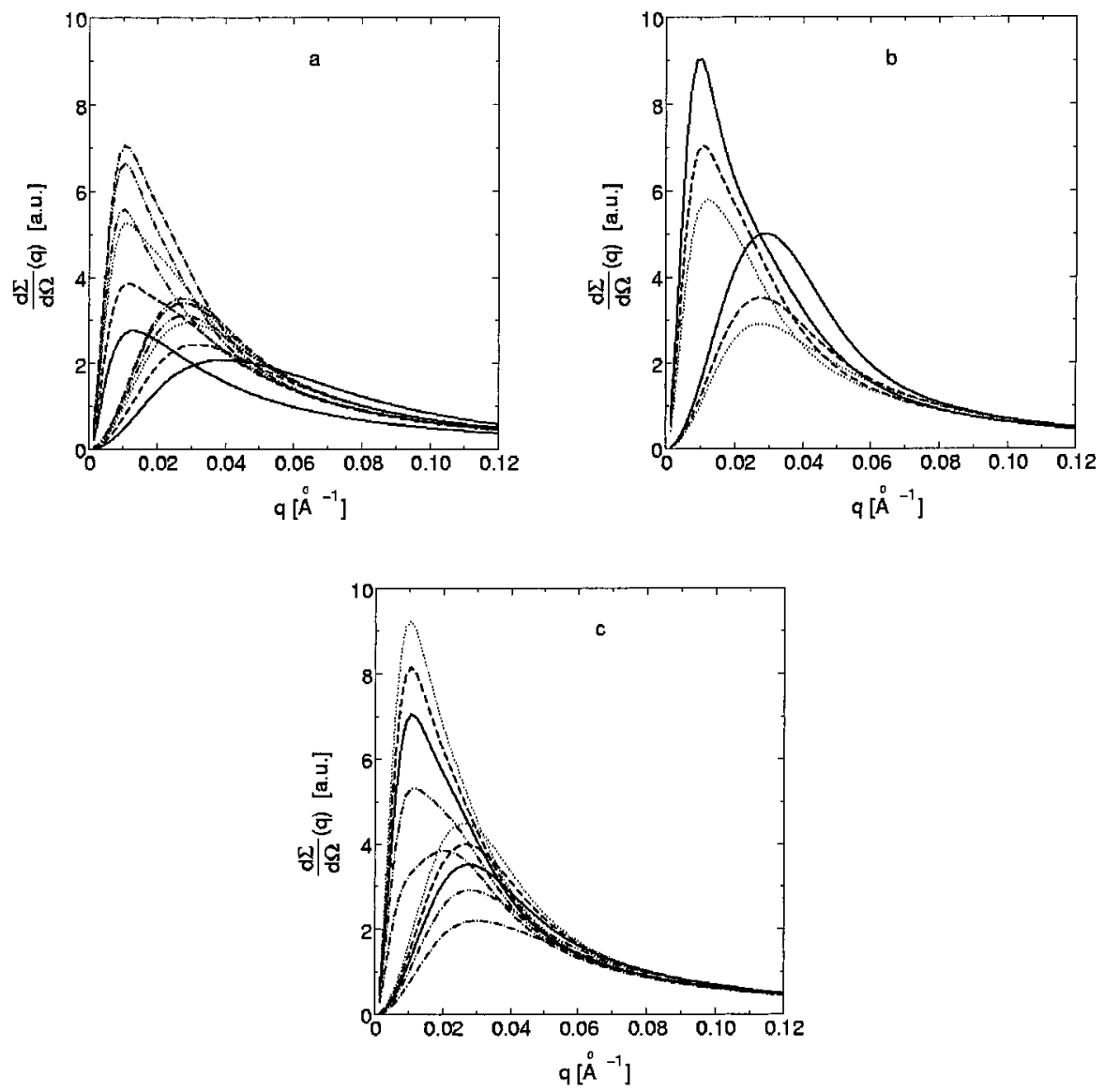

Figure 7. Scattering curves obtained by the "quenched-RPA" theory in the directions parallel (peak at lower q values) and perpendicular (peak at higher $q$ values) to the strain, calculated for $\lambda=3$ and $R_{\text {g,tip }}=32 \AA$. (a) Influence of $x$ at $d_{0}=38 \AA$, no branch point withdrawal and full arm retraction, for $\mathrm{x}=0.1(-) ; \mathrm{x}=0.15(-) ; \mathrm{x}=0.2(\cdots) ; \mathrm{x}=0.3(-\cdots) ; \mathrm{x}=0.4(-\cdots-) ; \mathrm{x}=$ $0.5(-\cdots-)$. (b) Influence of $d_{0}$, at $x=0.3$, no branch point withdrawal, full arm retraction, for $d_{0}=30 \AA(-)$, $\mathrm{d}_{0}=38 \AA(--)$, and $\mathrm{d}_{0}=44 \AA(\cdots)$. (c) Influence of branch point withdrawal and arm retraction at $\mathrm{x}=0.3, \mathrm{~d}_{0}=38 \AA$, for no branch point withdrawal and full arm retraction (-); one tube diameter withdrawal and full arm retraction (- -); two tube diameters withdrawal and full arm retraction $(\cdots)$; no branch point withdrawal and no arm retraction $(-\cdot-)$; no branch point withdrawal and arm retracted halfway $(-\cdots-)$.

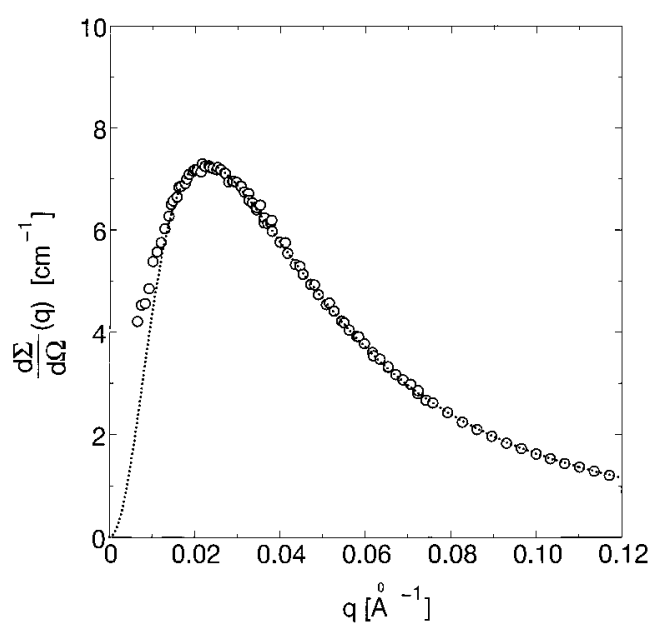

Figure 8. Experimental radially averaged isotropic coherent scattering intensity at $25{ }^{\circ} \mathrm{C}$ (circles) and annealed RPA structure factor (dotted line).

origin. Furthermore, a new feature appears: clearly, four off-axis peaks are present for the two highest annealing times which make an angle of about $45^{\circ}$ to the stretching axis. This feature is not reproduced by the tube model theory described bel ow and suggests that an analysis along principal axes is not sufficient (although we will attempt such an analysis below to ill ustrate further the discrepancies between theory and experiment!). From the scattering vector this corresponds to a length scale of $2 \pi / q \approx 400 \pm 50 \AA$, which is of the same order of magnitude as the mean end-to-end distance of the $\mathrm{H}$-polymer. The four peaks here are of a different orientation to the four-point "shamrock" pattern observed by Hayes et al. ${ }^{10}$ during the temporal transition from lozenge to butterfly patterns. Their orientation is similar to those seen by Rharbi et al. ${ }^{32}$ in scattering from a filled elastomer, but the explanation in that case (relative shearing motion of the hard filler particles) is not appropriate in the present soft system.

\section{Comparison of SANS with Theory}

The calculation of scattering patterns from a stretched melt of partially labeled $\mathrm{H}$-polymers, using the description of the tube model above, is far from straightforward. One aspect of the physics that must be addressed consistently is the statistical description of relaxed and unrelaxed sections of chains. The unrel axed inner chain sections are confined to a tube, which is anisotropically distributed and, we assume, affinely deformed from the isotropic melt. On the time scale of the experiment, these chain sections are quenched in a nonequilibrium configuration. The relaxed outer sections of chains are anneal ed on the time scale of the experiment and would be isotropic if there were no excluded-volume interactions. However, there are excluded-vol ume interactions 
with the unrelaxed chain sections which may induce anisotropy in the "relaxed" chain material. ${ }^{19}$

One of us has developed a scattering theory, based on the random phase approximation, to deal with these quenched and annealed variables. ${ }^{19}$ This makes use of two facts: (i) the exponential distribution of relaxation times in branched polymers enables a clear distinction to be drawn between relaxed (anneal ed) and unrelaxed (quenched) chain variables at a given time scale, and (ii) there are correlations between the quenched tube variables, due to the fact that they are affinely deformed from the isotropic melt, where correlations due to excluded-volume interactions are present.

Full details of this calculation may be found elsewhere; ${ }^{19}$ we summarize only the main results in the appendix. The variables required in the tube model calculation are as follows:

(i) The fraction of each chain which is contained in the cross-bar and the fraction of arms which are deuterium-labeled. These two variables are fixed by the reaction chemistry.

(ii) The monomer step length for Gaussian chain statistics. This can be measured in terms of a radius of gyration parameter by a scattering experiment on an unstretched $\mathrm{H}$-polymer sample.

(iii) The tube diameter, used in the Warner-E dwards tube model, can be obtained by fitting to the linear rheological response of the polymers. It may be scaled to account for tube dilation by constraint release, as in eq 2.

(iv) The fraction of the arms that have relaxed at a given relaxation time. This may be taken as a fixed parameter from tube model calculations on the linear rheology (given in Table 1).

(v) The degree of retraction of the arms. The experimental time scale is always longer than the arms Rouse relaxation time, so the arms should have retracted to their equilibrium tube length.

(vi) The degree of branch point withdrawal. Ideally, there would be no branch point withdrawal at stretches less than $\lambda=4$, but (as described above) there may be some branch point withdrawal due to local displacement of the branch point within the smooth elastic potential minimum of the tube branch point site. This branch point withdrawal must be on the order of magnitude of a tube diameter (note that the tube diameter can increase with time, due to tube dilation). The time scale for branch point withdrawal is about the Rouse time of the whole polymer, estimated as $0.04 \mathrm{~s}$. Since this is on the time scale of some of the experiments, we are free to choose different degrees of branch point withdrawal for different experimental times, subject to the constraint that the degree of withdrawal must increase with time.

It is clear from the above that, while there are many variables in the model, all of them are either fixed or would be strongly constrained by physical considerations respecting the tube model. Comparisons of this theory with scattering experiments thus constitute a strong test of the tube model, and any discrepancies may be regarded as an indication of deficiencies in the version of the tube model used, provided the behavior of the sample is simple.

One would hope, then, to be able to take the parameters for the calculation straight from the rheological characterization and use them to predict the scattering patterns of the experiment. As will be described below, we found this ideal scenario to be far from reality.

Nevertheless, so that the reader can judge the sensitivity of the theory to different parameters, we reproduce in Figure 7 sets of scattering curves averaged over $\pm 10^{\circ}$ from the directions parallel and perpendicular to a fixed stretch of $\lambda=3$. We take the same radius of gyration as is measured bel ow for the isotropic sample. In each plot, a single parameter is varied away from a base set of parameters consisting of no branch point withdrawal, full arm retraction, and $\mathrm{x}=0.3$ and $\mathrm{d}_{0}=$ $38 \AA$ (note that here we fix the tube diameter after dynamic dilution has been applied, rather than varying it as $x$ varies according to eq 2 ). In Figure $7 a$ we show how the scattering varies as $x$ changes from 0.1 to 0.5 . It can be seen that both the parallel and perpendicular peaks increase dramatically up until $x \approx 0.3$ and then decrease again as $x$ increases further. The high- $q$ scattering becomes monotonically more isotropic as $x$ increases. In Figure 7b we show how the scattering changes as $d_{0}$ changes from 30 to $44 \AA$. As the tube diameter increases, the parallel and perpendicular peaks both decrease in height, and the high-q scattering becomes more isotropic. For larger or smaller values of $x$, the same trends are evident but not so pronounced in the low-q region. In Figure 7c we show how the scattering varies, first as the arms retract from their fully extended lengths and then as the branch point withdraws. It can be seen that both retractions lead to substantial increases in the peak heights parallel and perpendicular to the stretch. Again, this effect is less pronounced for larger or smaller values of $x$ (see discussion and text bel ow for Figure 9 and Figure 11), but the trend is the same.

It is clear that the theory predicts some anisotropy in peak height. The mechanism for this can be understood as follows. In the limit of tight constraints (very small tube diameters) the perpendicular peak would lie at quite high wavevectors and be of similar magnitude to the parallel peak. However, such wavevectors correspond to length scales on which the polymers are relaxed and the scattering tends to isotropy. The highwavevector component of the perpendicular peak is "washed out" as the chains can move and destroy any correlations on these length scales; all that remains is the low-wavevector "tail" of the peak, which is smaller in magnitude than the parallel peak. Note that the anisotropy predicted within this theoretical framework has nothing to do with the elastic inhomogeneities proposed to explain butterfly scattering patterns; such inhomogeneities are not included within the present theory.

It is also clear that the theory predicts significant enhancement of the peak heights for particular choices of parameters. The requirements for such an enhancement in this theory appear to be (i) some retraction occurring in the tube, either in the arms or in branch point withdrawal, and (ii) some, but not too much, relaxation of the arms. The retraction correlates more monomers within a smaller volume (i.e., within a shorter section of tube) and hence provides the potential for a larger variation in composition for scattering. However, to realize this variation in composition to the largest possible extent, there must be relatively mobile sections of chain which are affected by excluded-vol ume interactions with the retracted chain and which provide scattering contrast. This function is filled by the relax- 

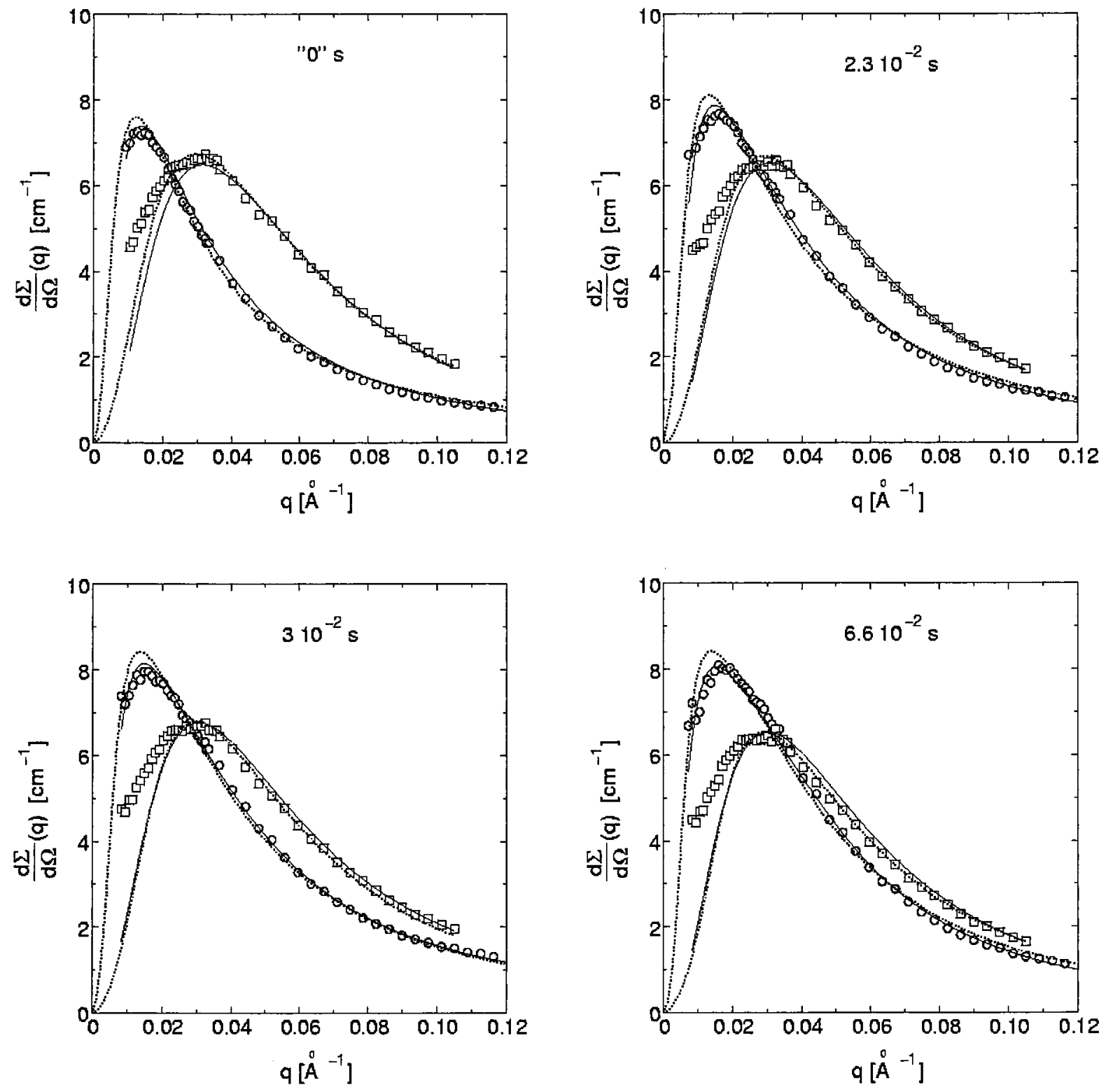

Figure 9. Experimental coherent scattering intensity al ong the directions parallel to the deformation (circles) and perpendicular to the deformation (squares) at $-85^{\circ} \mathrm{C}$, compared with both theoretical approaches (solid lines, "quenched-RPA" approach; dotted lines, "factorized-RPA" approach) for the sample strained to $\lambda=2$ and after annealing times " 0 ", $2.3 \times 10^{-2}, 3.0 \times 10^{-2}$, and 6.6 $\times 10^{-2} \mathrm{~s}$ at $25^{\circ} \mathrm{C}$.

ation of the labeled arm tips. If $x$ is too small, then not enough of the labeled portion is relaxed to be affected. If $\mathrm{x}$ is too large, then there is less retracted chain in tubes, so the effect is diminished.

A final point is that, although it is possible to obtain significant increases in peak heights within the theory, it is more difficult to obtain increases in peak ani sotropy; increases in parallel peak height tend to be accompanied by increases in perpendicular peak height. Also, the tube diameter $\mathrm{d}_{0}$ is tied to relaxation of the arms via $\times$ (see eq 2). Since we must fit parallel and perpendicular scattering with the same set of parameters (actually we should fit the whole scattering pattern) and since the variation of parameters for a given time series must be consistent, in practice there is not much flexibility in the fitting procedure.

In addition to the above theory, we also tested a simpler expression for the scattering, which had been used previously in the description of triblock networks (again, details are given in the appendix). Because of the fact that in this expression the structure function for the quenched disorder case is approximated by a product of the intra- and interchains correlation functions (see appendix), the incompressibility condition is only fulfilled for the initial relaxation times. Despite this, this approach yields similar results to the more detailed theory above, especially in the limit of strong constraints. While it is not possible to include all of the above effects in this simpler expression, it is possible to include tube dilation and arm relaxation but not arm retraction or branch point withdrawal.

5.1. Isotropic Melt. Figure 8 presents the radially averaged isotropic coherent scattering intensity of the $\mathrm{H}$-polymer at $\mathrm{T}=25{ }^{\circ} \mathrm{C}$. The fit through the data is given by the standard annealed RPA formula. From separate experiments at $\mathrm{T}=-30{ }^{\circ} \mathrm{C}$ and $\mathrm{T}=-85^{\circ} \mathrm{C}$ (not shown) in which the peak height was carefully checked, it was concluded that the Flory-Huggins parameter $\chi$ could be neglected completely. The experimental value obtained for the radius of gyration of the 


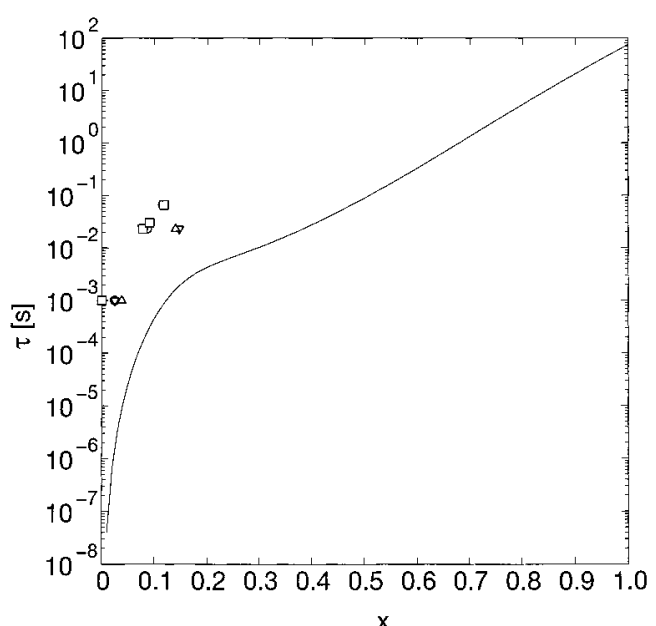

Figure 10. Theoretical annealing time $\tau$ as a function of $\mathrm{x}$, the abscissa al ong one $\operatorname{arm}(\mathrm{x}=0$ at the branch point) obtained from linear measurement in oscillatory shear (solid line) compared to the results obtained by both theoretical approaches for the SANS results: strain $\lambda=2$, "quenched-RPA" approach (circles) and "factorized-RPA" approach (squares). strain $\lambda=3$, "quenched-RPA" approach (triangles down) and "factorized-RPA" approach (triangles up).

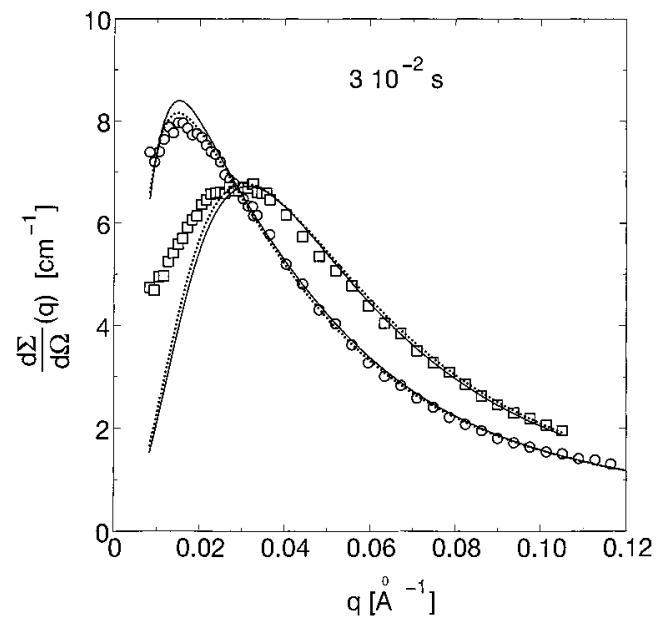

Figure 11. Experimental coherent scattering intensity along the directions parallel to the deformation (circles) and perpendicular to the deformation (squares) at $-85^{\circ} \mathrm{C}$, compared with the "quenched-RPA" approach, assuming no arm retraction (dotted lines) and full arm retraction (solid lines) for the sample strained to $\lambda=2$ and after $3 \times 10^{-2} \mathrm{~s}$ annealing time at $25^{\circ} \mathrm{C}$.

deuterated tip, $R_{\text {g,tip }}=32 \pm 2 \AA$, is in good agreement with the unperturbed radius of gyration expected from the power law $R_{g}=0.315 \mathrm{M}^{0.5} .{ }^{33}$ The lower q part in the left wing of the correlation peak is clearly the signature of polydispersity in the number of components due to synthesis and coupling of the respective components into the $\mathrm{H}$-architecture. It can be shown not to interfere in the analysis of the right wing and is not corrected for since a detailed description of these polymers is lacking.

5.2. $\lambda=\mathbf{2}$ and Relaxation. Data along both principal axes of deformation are summarized in Figure 9 along with theoretical fits to the scattering. Here, solid and dotted lines refer to both suggested approaches of RPA structure factors (the full "quenched-constraint" theory and "factorized" approximation, respectively). The fits appear very good at first sight, and the parameters obtained by both theories are almost identical (see Table 1 ). But this must be taken in the context of the comparison of the fitting parameters with those predicted from the rheological characterization. It was not by any means possible to describe the data using parameters directly from the rheol ogy. The parameters used differ from those expected from rheol ogy as follows:

1. The undiluted tube diameter was much smaller than expected. We obtained values of $d_{00}=33 \pm 3 \AA$ (the actual tube diameter was "dilated" as the arms relaxed, via eq 2 ), whereas $\mathrm{PI}$ networks yield $\mathrm{d}_{00}=42$ $\pm 2 \AA$. 34 The rheological characterization used an entanglement molecular weight of $M_{e}=4400 \mathrm{~g} \mathrm{~mol}^{-1}$, corresponding to a tube diameter of $51 \AA$. If larger tube diameters are used, then the high wavevector scattering is predicted to be much too isotropic. The difference may be to do with the discrepancy between the WarnerEdwards picture of the tube (a series of harmonic confining potentials) and the actual confining tube.

2. The fraction, $x$, of the arms relaxed used to fit the SANS was much smaller than that expected from rheology. Table 1 and Figure 10 summarized this difference for each of the probed relaxation times. Note, for example, that at $6.6 \times 10^{-2} \mathrm{~s}$ relaxation we expect roughly half the arm to have relaxed, whereas the fit to SANS indicates only a fraction $x=0.12$ has relaxed (corresponding to a relaxation time of less than $10^{-3} \mathrm{~s}$ ). The small value of $x$ used is required to retain the anisotropy at high wavevectors, the ratio of the perpendicular and parallel peak heights, and their shape.

3. The best fits were obtained if no arm retraction (or branch point withdrawal) was assumed. Note that, given the predicted arm retraction time of about 0.004 $\mathrm{s}$, we would expect a partial retraction of the arm in even the earliest (" $0 \mathrm{~s}$ ") data set, with total arm retraction in the later data sets. We might even expect some branch point withdrawal occurring in the later data sets. For the sake of comparison, Figure 11 compares the experimental data for the $3.0 \times 10^{-2} \mathrm{~s}$ relaxation with theoretical curves with and without arm retraction. For this set of parameters, in particular the small value of $x$, there is not a great deal of difference between the two, but the curve without retraction is clearly the better. Note the contrast between the effect of retraction at this small value of $x$ and that seen above in Figure 7c.

The last two discrepancies are indicative of a polymer melt that is much less relaxed than was expected. Indeed, the indication of no arm retraction is consistent (in terms of the effective relaxation time) with the degree of arm relaxation. We must seek an explanation of these observations.

5.3. $\lambda=3$ and Relaxation. Data along both principal axes of deformation are summarized in Figure 12 al ong with attempted theoretical fits to the scattering. Reasonable fits could be obtained for the earliest two relaxation times, although the fitted tube diameter is again smaller than we would expect from rheology and the arm relaxation variable $x$ is again smaller than expected (as summarized in Table 1 and Figure 10). In the case of the $2.3 \times 10^{-2} \mathrm{~s}$ data, the fit shown includes the expected full arm retraction, but no branch point withdrawal. (This is in contrast to the best fit obtained for the $\lambda=2$ data at similar relaxation time.)

The most striking feature in the later two relaxation times is that the peak parallel to the stretch keeps on growing as the relaxation time increases. Although this is a qualitative prediction of the effects of arm relaxation and retraction and of branch point withdrawal, the 

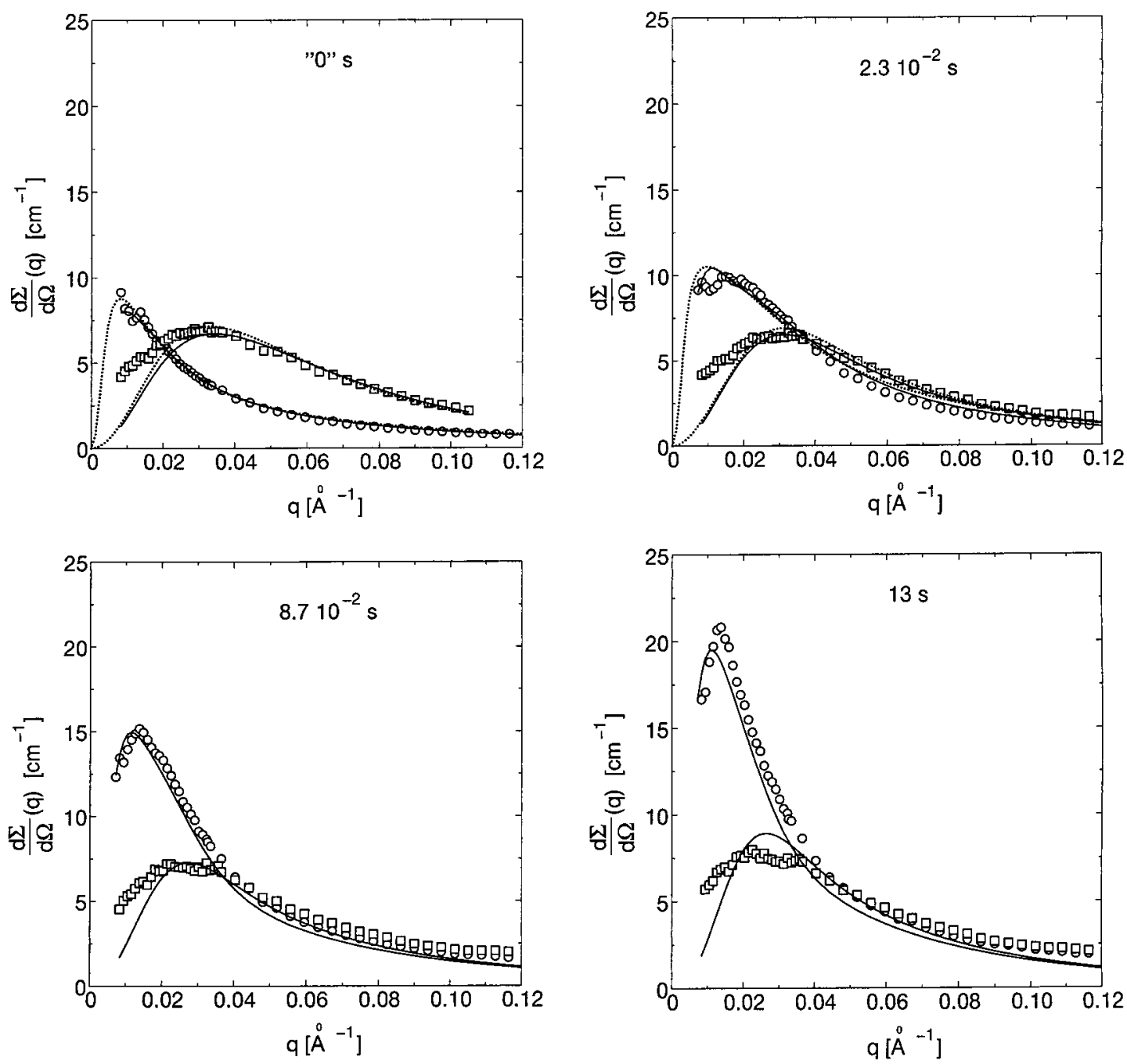

Figure 12. Experimental coherent scattering intensity al ong the directions parallel to the deformation (circles) and perpendicular to the deformation (squares) at $-85^{\circ} \mathrm{C}$, compared with both theoretical approaches (solid lines, "quenched-RPA" approach; dotted lines, "factorized-RPA" approach) for the sample strained to $\lambda=3$ and after annealing times " 0 ", $2.3 \times 10^{-2}, 8.7 \times 10^{-2}$, and 13 $\mathrm{s}$ at $25^{\circ} \mathrm{C}$.

anisotropy of the experimental signal defies explanation within our current framework. A feature of the detailed theory is that it predicts an increase in the scattering parallel to the stretch as the arm relaxation variable, $x$, increases up until $x \approx 0.3$. For higher values of $x$ the predicted parallel scattering peak decreases again. (In the experiment, it is only seen to grow!) This parallel peak is strongly enhanced by arm retraction and branch point withdrawal. So, to try to emulate the experimental results with the theory, one is forced to use values of $x$ around 0.3 , full arm retraction, and varying amounts of branch point withdrawal. In this way, a fit can be obtained for the scattering parallel and perpendicular to the stretch in the $8.7 \times 10^{-2} \mathrm{~s}$ data, and the high parallel peak in the $13 \mathrm{~s}$ data can be approached (but not matched). However, such a fitting procedure is (obviously) far from satisfactory. Again, parameters are shown in Table 1, and the values of $x$ used remain on the small side when compared to rheology. Bearing in mind that there are features in the 2-d scattering (the four-point pattern) not reproduced at all by the theory, it is safe to conclude that the theory is lacking in its description of the experimental data in this late time regime. It is regrettable that due to time constraints no data were taken in this later time regime for $\lambda=2$.

\section{Discussion and Conclusion}

Our attempts at fitting the experimental scattering data with the current tube model scattering theory ran into serious problems, which can be summarized as follows. For $\lambda=2$, the melt appeared to be much less relaxed than expected (less arm relaxation and no arm retraction). This discrepancy in degree of relaxation was to some extent reflected in the $\lambda=3$ data. Furthermore, the later relaxation times produced an increased scattering parallel to the stretch (much larger than predicted by theory) and a four-point pattern in the 2-d scattering (not predicted at all by theory). For this larger stretch, the best fits from theory did require retraction of the arms and branch point withdrawal; otherwise, the large parallel peak was not predicted at all. Earlier scattering experiments ${ }^{18}$ on the same sample, while lacking the control of relaxation time in this present experiment, produced a similarly large increase in scattering parallel to the stretch but did not show any evidence of a four-point pattern.

In the absence of further evidence, we are forced to speculate on the origin of these discrepancies. The most obvious explanation for the lack of relaxation in the $\lambda$ $=2$ data sets is that there is some error in the assigned relaxation time. The experimental relaxation times are 
based on the WLF shifting procedure and are thus sensitive to the temperature control in the experiment. We believe we have eliminated the possibility of an error in the temperature measurement by checking the temperature both in and around the sample during a test run: the temperatures agreed to fractions of a degree. On this basis, we cannot account for the apparently much sl ower relaxation of the sample by citing an error in the measured temperature. Remaining possibilities include a breakdown in the WLF shifting for the strained sample, or merely peculiarities of the sample, but this requires further testing by experiment.

For the later time data at $\lambda=3$, there are a variety of possible deficiencies in the tube model used for the scattering calculation, some of which have been highlighted previously ${ }^{19-}$-here we shall only list them briefly.

1. The calculation preaveraged the retraction of the polymer arms and the branch point withdrawal, assuming that all polymers retract by the same amount. This is certainly not the case when chains are short and contain only a few (of order 10) tube lengths.

2. The calculation did not include any of the recently suggested modifications to the tube model in the nonlinear regime which we listed in the Introduction and repeat here: convective constraint release, ${ }^{20-22}$ tube dilation and distortion, ${ }^{23-25}$ nonaffine entanglement deformation from local chain force balance, ${ }^{26}$ and el astic inhomogeneities in the entanglement network. ${ }^{12,13}$

3. The calculation assumed that the Warner-Edwards localizing potentials remain isotropic with the stretch-it has been speculated in networks that these should couple to the stretch.

It is certainly necessary to evaluate the effect of each of these omissions on the scattering calculation, and it is possible to see how some of these might give rise to increased scattering parallel to the stretch. In particular, elastic inhomogeneities in the entanglement network are known to produce this effect in blends of long and short polymers, and gels (see e.g. refs 9, 10, and 35). The scattering patterns here retain the "correlation hol e" structure of a block copolymer melt and so are not the same as the so-called "butterfly" patterns; nevertheless, the origin of the anisotropy could be the same. We note also that the earlier scattering experiments of Boué et al. on linear chains, 7,8 which share some of the features seen here (no strong evidence for retraction, apparently small tube diameter), have not been reanalyzed in the light of many of the modifications to the tube theory listed above.

However, while it is possible to see how some of the above mechanisms give rise to increased scattering parallel to the stretch, it is not a priori obvious that any will produce the four-point pattern. Similar four-point patterns were observed recently in scattering from stretched filled elastomers, ${ }^{32}$ but the proposed origin of the pattern in that experiment (relative shearing of hard nanoparticles) would not appear to be a canditate for the present experiment.

Acknowledgment. The authors thank M. Heiderich and $U$. Bünten for assistance at the SANS experiment at FZ J ülich. We are indebted to $\mathrm{H}$. Feil bach and $\mathrm{G}$. Vehres of FZ J ülich for the construction of the microprocessor controlled strain rig. This work was performed with financial support in the framework of The BRITE EURAM project BR-97-4248, "LCB Polyolefins".

\section{Appendix. SANS Modeling}

Calculation of the scattering pattern from a stretched melt of partially labeled $\mathrm{H}$-polymers, using the description of the tube model above, is far from straightforward. One aspect of the physics that must be addressed consistently is the statistical description of relaxed and unrelaxed sections of chains. The unrel axed inner chain sections are confined to a tube, which is anisotropically distributed and, we assume, affinely deformed from the isotropic melt. On the time scale of the experiment, these chain sections are partially quenched in a nonequilibrium configuration. The relaxed outer sections of chains are annealed on the time scale of the experiment and would be isotropic if there were no excludedvolume interactions. However, there are excludedvolume interactions with the unrelaxed chain sections which may induce anisotropy in the "relaxed" chain material. Further, the dynamic asymmetry due to the different mobility of arm and cross-bar segments may cause a more complicated behavior at late stage of relaxation.

One of us has developed a scattering theory, based on the random phase approximation, to deal with these quenched and annealed variables. This makes use of two facts: (i) the exponential distribution of relaxation times in branched polymers enables a clear distinction to be drawn between relaxed (annealed) and unrelaxed (quenched) chain variables at a given time scale, and (ii) there are correlations between the quenched tube variables, due to the fact that they are affinely deformed from the isotropic melt, where correlations due to excluded-volume interactions were present.

Full details of this RPA calculation may be found el sewhere; ${ }^{19}$ here only the main results are summarized. The calculation accounts for excluded-volume interactions both before the stretch (these lead to correlations between quenched tube variables) and after the stretch (giving incompressibility in the stretched melt) via the definition of polymer density fiel ds before and after the stretch. After the stretch the position of monomer I on chain $\alpha$ is $\mathbf{r}_{1}^{\alpha}$ and we define Fourier transformed densities of the labeled (A) monomers and unlabel ed (B) monomers as

$$
\begin{gathered}
\rho_{\mathbf{q}}^{A}=\sum_{\alpha, l} y_{1}^{\alpha} \exp \left(i \mathbf{q} \cdot \mathbf{r}_{1}^{\alpha}\right) \\
\rho_{\mathbf{q}}^{\mathrm{B}}=\sum_{\alpha, l}\left(1-y_{1}^{\alpha}\right) \exp \left(\mathbf{i q} \cdot \mathbf{r}_{1}^{\alpha}\right)
\end{gathered}
$$

where $y_{1}^{\alpha}=1$ if the monomer is labeled and $y_{1}^{\alpha}=0$ otherwise. I nitially, the position of monomer I on chain $\alpha$ is $\mathbf{x}_{1}^{\alpha}$, and we define the total monomer density

$$
\varphi_{\mathbf{q}}=\sum_{\alpha, 1} \exp \left(\mathbf{i q} \cdot \mathbf{E} \cdot \mathbf{x}_{1}^{\alpha}\right)
$$

Notice that the sum in $\varphi_{\mathbf{q}}$ is over all A and B monomers. We include the strain tensor $\mathbf{E}$ in this definition to bring the $\mathbf{x}_{1}^{\alpha}$ into the same space as the $\mathbf{r}_{1}^{\alpha}$, thus avoiding problems with translational symmetry in later averages.

The formal RPA result for the scattering function subject to incompressibility in the melt after the stretch and quenched tube variables is 


$$
\begin{aligned}
& S(\mathbf{q})= \frac{T_{\mathbf{q}}^{A A} T_{\mathbf{q}}^{\mathrm{BB}}-\left(T_{\mathbf{q}}^{\mathrm{AB}}\right)^{2}}{\mathrm{~T}_{\mathbf{q}}^{\mathrm{AA}}+\mathrm{T}_{\mathbf{q}}^{\mathrm{BB}}+2 \mathrm{~T}_{\mathbf{q}}^{\mathrm{AB}}+} \\
& \frac{\Delta_{\mathbf{q}}^{\mathrm{AA}}\left(\mathrm{T}_{\mathbf{q}}^{\mathrm{BB}}+\mathrm{T}_{\mathbf{q}}^{\mathrm{AB}}\right)^{2}+\Delta_{\mathbf{q}}^{\mathrm{BB}}\left(\mathrm{T}_{\mathbf{q}}^{\mathrm{AA}}+\mathrm{T}_{\mathbf{q}}^{\mathrm{AB}}\right)^{2}}{\left(\mathrm{~T}_{\mathbf{q}}^{\mathrm{AA}}+\mathrm{T}_{\mathbf{q}}^{\mathrm{BB}}+2 \mathrm{~T}_{\mathbf{q}}^{\mathrm{AB}}\right)^{2}} \\
& \frac{2 \Delta_{\mathbf{q}}^{\mathrm{AB}}\left(\mathrm{T}_{\mathbf{q}}^{\mathrm{AA}}+\mathrm{T}_{\mathbf{q}}^{\mathrm{AB}}\right)\left(\mathrm{T}_{\mathbf{q}}^{\mathrm{BB}}+\mathrm{T}_{\mathbf{q}}^{\mathrm{AB}}\right)}{\left(\mathrm{T}_{\mathbf{q}}^{\mathrm{AA}}+\mathrm{T}_{\mathbf{q}}^{\mathrm{BB}}+2 \mathrm{~T}_{\mathbf{q}}^{\mathrm{AB}}\right)^{2}}
\end{aligned}
$$

There are two types of structure factor which must be calculated in eq 6 . The first is of type

$$
\Delta_{\mathbf{q}}^{\mathrm{J}}=\overline{\left\langle\rho_{\mathbf{q}}^{\prime}\right\rangle_{0}\left\langle\rho_{\mathbf{q}}^{J}\right\rangle_{0}}
$$

where the labels I and J can each be either A or B. The annealed averages are denoted by brackets and have been calculated in the absence of chain-chain interactions (denoted by the subscript 0 ), but subject to quenched tube variables. The average over these quenched variables (denoted by overbar) must include correlations between the tubes due to incompressibility in the melt prior to the stretch. The structure factors of type $\Delta_{\mathbf{q}}^{\mathrm{IJ}}$ are related to the nonzero fluctuations of the concentrations due to quenched variables.

The second type of structure factor is

$$
T_{\mathbf{q}}^{l J}=\overline{\left\langle\rho_{\mathbf{q}}^{\prime} \rho_{-\mathbf{q}}^{J}\right\rangle_{0}-\left\langle\rho_{\mathbf{q}}^{\prime}\right\rangle_{0}\left\langle\rho_{\mathbf{q}}^{J}\right\rangle_{0}}
$$

which is related to fluctuations about the mean of the frozen-in concentration fluctuations. The subscripts 0 in eq 8 denote again that the annealed averages have to be performed subjected to tube constraints but without chain-chain interactions.

These two types of structure factor may be written in terms of single chain structure factors, subjected only to tube constraints in deformed tubes. The results of a RPA treatment of tube-tube correlations are

$$
T_{\mathbf{q}}^{I J}=T_{0 \mathbf{q}}^{I J}={\overline{\left\langle\rho_{\mathbf{q}}^{\prime} \rho_{-\mathbf{q}}^{J}\right\rangle_{0}-\left\langle\rho_{\mathbf{q}}^{\prime}\right\rangle_{0}\left\langle\rho_{\mathbf{q}}^{J}\right\rangle_{0}}}_{0}^{0}
$$

where the superscript 0 denotes the absence of tubetube correlations in the quenched average. The correlation function $\Delta_{\mathbf{q}}^{\mathrm{IJ}}$ is given by

$$
\Delta_{\mathbf{q}}^{J J}=\Delta_{0 \mathbf{q}}^{J J}-\frac{D_{\mathbf{q}}^{\prime} D_{\mathbf{q}}^{J}}{S_{\mathbf{q}}^{\text {tot }}}
$$

with the following single chain correlation functions

$$
\begin{aligned}
& \Delta_{0 \mathbf{q}}^{\mathrm{J}}={\overline{\left\langle\rho_{\mathbf{q}}\right\rangle_{0}\left\langle\rho_{\mathbf{q}}^{J}\right\rangle_{0}}}^{0} \\
& \mathrm{D}_{\mathbf{q}}^{\mathrm{I}}=\overline{\left\langle\rho_{\mathbf{q}}^{\mathrm{I}}\right\rangle_{0}\left\langle\varphi_{\mathbf{q}}\right\rangle_{0}}{ }^{0} \\
& \mathrm{~S}_{\mathbf{q}}^{\text {tot }}=\overline{\left\langle\varphi_{\mathbf{q}} \varphi_{-\mathbf{q}}\right\rangle_{0}}{ }^{0}
\end{aligned}
$$

where again the superscript 0 denotes the absence of tube-tube correlations in the quenched average.

Explicit expressions for the correlation functions in eqs 9 and 13 were obtained within the WarnerE dwards model where the chains are restricted to the space with the tube radius $d_{0}$ around the tube axis. ${ }^{36}$
A more intuitive and much simpler treatment of the scattering in undeformed and deformed melts follows the approach which was used in the description of permanent networks of long-labeled paths ${ }^{34}$ or centerlabeled chains. ${ }^{29}$ This approach is expected to be applicable with good accuracy for the scattering properties immediately after deformation because in the case of rapidly applied deformations a very network-like behavior should occur. For a description of the scattering during the arm relaxation process, the time evolution of correlations in the system must be taken into account approximately by the following considerations:

(i) During the arm relaxation process the interchain correlation is unchanged since it reflects the center of gravity of the chains in the system.

(ii) The arm relaxation is described by progressively replacing the outer part of the deformed arm by an undeformed one, the inner one still fully deformed, which gives rise to a "block" structure in labeling and strain as well.

(iii) An increase of $d_{0}$ due to dynamic dilution can be considered.

This could be conveniently described by a factorization in intra- and interchain contributions. The constraints due to neighboring chain configurations are taken independent of long-range correlations between chains in this approximation. This yields mathematically, to be compared to eq 6 ,

$$
S(q)=\frac{S_{R P A}(\lambda)}{S_{0}^{A A}(\lambda)} \tilde{S}^{A A}\left(q, \lambda, d_{0}\right)
$$

where $\mathrm{S}_{0}^{\mathrm{AA}}$ and $\tilde{\mathrm{S}}^{\mathrm{AA}}$ are defined as respectively ideal "phantom" chain structure factor and Warner-Edwards model scattering functions as before.

For narrow tubes i.e., $d_{0} \ll R_{g}$, eq 6 is converted to

$$
\mathrm{S}_{\mathrm{RPA}}(\mathbf{q})=\frac{\mathrm{S}_{0}^{\mathrm{AA}}(\mathbf{q} \cdot \mathbf{E}) \mathrm{S}_{0}^{\mathrm{BB}}(\mathbf{q} \cdot \mathbf{E})-\mathrm{S}_{0}^{\mathrm{AB}}(\mathbf{q} \cdot \mathbf{E})^{2}}{\mathrm{~S}_{0}^{\mathrm{AA}}(\mathbf{q} \cdot \mathbf{E})+\mathrm{S}_{0}^{\mathrm{BB}}(\mathbf{q} \cdot \mathbf{E})+2 \mathrm{~S}_{0}^{\mathrm{AB}}(\mathbf{q} \cdot \mathbf{E})}
$$

which corresponds to the standard RPA result for a melt, subjected to an affine displacement of all coordinates. In this limit, eqs $6,9,10,13$, and 15 directly give the scattering properties immediately after a fast deformation.

The AA superscript assume all combinations of different tip pairs along the $\mathrm{H}$-polymer which belong to either a deformed segment or an outerlying isotropically relaxed block. Note that relaxation effects as well as tube dilution enter the intrachain term only. Therefore, selective information like unrelaxed cross-bar configurations can be input. Details about the structure of the correlation functions in eq 14 are given in ref 29. The calculation of the bare structure factors in eq 15 is straightforward using the elementary Debye-like contributions (see e.g. in ref 37). The isotropic melt scattering data are described identically in both approaches for the investigated special $\mathrm{H}$-architecture.

\section{References and Notes}

(1) Doi, M.; Edwards, S. F. In TheTheory of Polymer Dynamics; Clarendon Press: Oxford, 1986.

(2) Osaki, K.; Nishizawa, K.; Kurata, M. Macromolecules 1982, 15, 1068-1071.

(3) Milner, S. T.; McLeish, T. C. B. Phys. Rev. Lett. 1998, 81, $725-728$. 
(4) Higgins, J . S.; Benoit, H. In Polymers and Neutron Scattering; Clarendon Press: Oxford, 1994.

(5) Schleger, P.; Farago, B.; Lartigue, C.; Kollmar, A.; Richter, D. Phys. Rev. Lett. 1998, 81, 124-127.

(6) Pütz, M.; Kremer, K.; Grest, G. S. Europhys. Lett. 2000, 49, $735-741$.

(7) Boué, F.; Nierlich, M.; J annink, G.; Ball, R. C. J . Phys. (Paris) 1982, 43, 137-148. Boué, F.; Nierlich, M.; J annink, G.; Ball, R. C. J . Phys., Lett. 1982, 43, L585-L591. Boué, F.; Nierlich, M.; J annink, G.; Ball, R. C. J . Phys., Lett. 1982, 43, L593L600.

(8) Boué, F. Adv. Polym. Sci. 1987, 82, 47-101.

(9) Boué, F.; Bastide, J .; Buzier, M.; Lapp, A.; Herz, J .; Vilgis, T. A. Colloid Polym. Sci. 1991, 269, 195-216.

(10) Hayes, C.; Bokobza, L.; Boué, F.; Mendes, E.; Monnerie, L. Macromolecules 1996, 29, 5036-5041.

(11) Barea, J . L.; Muller, R.; Picot, C. In Polymer Motion in Dense Systems; Proceedings in Physics 29; Richter, D., Springer, T., Eds.; Springer-Verlag: Berlin, 1988; pp 86-92.

(12) Panyukov, S. V. J ETP 1992, 75, 347-352.

(13) Rouf, C.; Bastide, J .; Pujol, J .; Schosseler, F.; Munch, J . P. Phys. Rev. Lett. 1994, 73, 830-833.

(14) McLeish, T. C. B.; Larson, R. G. J . Rheol. 1998, 42, 81110.

(15) Inkson, N. J .; McLeish, T. C. B.; Harlen, O. G.; Groves, D. J . J. Rheol. 1999, 43, 873-896.

(16) Blackwell, R. J .; McLeish, T. C. B.; Harlen, O. G. J . Rheol. 2000, 44, 121-136

(17) Bick, D. K.; McLeish, T. C. B. Phys. Rev. Lett. 1996, 76, 25872590

(18) McLeish, T. C. B.; Allgaier, J .; Bick, D. K.; Bishko, G.; Biswas, P.; Blackwell, R.; Blottière, B.; Clarke, N.; Gibbs, B.; Groves, D. J .; Hakiki, A.; Heenan, R.; J ohnson, J . M.; Kant, R.; Read, D. J .; Young, R. N. Macromolecules 1999, 32, 67346758.

(19) Read, D. J . Eur. Phys. J . B 1999, 12, 431-449.
(20) Marrucci, G. J . Non-Newtonian Fluid Mech. 2000, 95, 369374

(21) Mead, D. W.; Larson, R. G.; Doi, M. Macromolecules 1998 31, 7895-7914.

(22) Milner, S. T.; McLeish, T. C. B.; Likhtman, A. E. J . Rheol. 2001, 45, 539-563.

(23) Wagner, M. H.; Bastien, P.; Ehrecke, P.; Kraft, M.; Huckmann, P.; Meissner, J . J . Non-Newtonian Fluid Mech. 1998, 79, 283-296.

(24) Öttinger, H. C. J . Non-Newtonian Fluid Mech. 2000, 89, 165185.

(25) Heinrich, G.; Straube, E.; Helmis, G. Adv. Polym. Sci. 1988 85, 33-87.

(26) Marrucci, G.; Greco, F.; I anniruberto, G. Rheol. Acta 2001 40, 98-103.

(27) Perny, S.; Allgaier, J .; Cho, D.; Lee, W.; Chang, T. Macromolecules 2001, 34, 5408-5415.

(28) Muller, R.; Pesce, J . J .; Picot, C. Macromolecules 1993, 26 , $4356-4362$

(29) Westermann, S.; Urban, V.; Pyckhout-Hintzen, W.; Richter D.; Straube, E. Macromol ecules 1996, 29, 6165-6174.

(30) Ferry, J . D. In Viscoel astic Properties of Polymers, 3rd ed.; J . Wiley \& Sons: New York, 1980.

(31) McLeish, T. C. B. Macromolecules 1988, 21, 1062-1070.

(32) Rharbi, Y.; Cabane, B.; Vacher, A.; J oanicot, M.; Boué, F. Europhys. Lett. 1999, 46, 472-478.

(33) Fetters, L. J .; Lohse, D. J .; Richter, D.; Witten, T. A.; Zirkel, A. Macromol ecules 1994, 27, 4639-4647.

(34) Straube, E.; Urban, V.; Pyckhout-Hintzen, W.; Richter, D. Glinka, C. J . Phys. Rev. Lett. 1995, 74, 4464-4467.

(35) Ramzi, A.; Zielinski, F.; Bastide, J .; Boué, F. Macromol ecules 1995, 28, 3570-3587.

(36) Warner, M.; Edwards, S. F. J . Phys. A 1978, 11, 1649-1655.

(37) Read, D. J . Macromol ecules 1998, 31, 899-911.

MA011923X 